\title{
Detection of small scale heterogeneities at the Inner Core Boundary
}

\author{
Anais Ibourichene ${ }^{\mathrm{a}, *}$, Barbara Romanowicz ${ }^{\mathrm{a}, \mathrm{b}, \mathrm{c}}$ \\ a Institut de Physique du Globe, 1 rue Jussieu, 75238 Paris Cedex 05, France \\ ${ }^{b}$ Collège de France, 11 Place Marcelin Berthelot, 75005 Paris, France \\ ${ }^{c}$ Berkeley Seismological Laboratory, 215 McCone Hall \# 4760, Berkeley, CA 94720, USA
}

\section{A R T I C L E I N F O}

\section{Keywords:}

ICB

Inner core

PKP(DF)

PKiKP

F-layer

\begin{abstract}
A B S T R A C T
The hemispherical pattern of seismic velocities in the uppermost inner core has been described at the global scale in many studies. In this paper, we investigate shorter scale heterogeneities in the vicinity of the Inner Core Boundary (ICB) using differential travel time and amplitude measurements between the pair of core phases PKP (DF) and PKiKP. Seismic events occurring close to Sumatra and recorded at USArray stations in the distance range $132-142^{\circ}$ are used to investigate the velocity and attenuation structure at the ICB and in the uppermost $100 \mathrm{~km}$ of the inner core beneath Eastern Russia.

In this distance range, $\mathrm{PKP}(\mathrm{DF})$ and PKiKP are difficult to separate due to their proximity in travel time. We present a new method based on the envelope of the seismic signal to identify them, following which we measure differential travel time residuals and amplitude ratios by comparison with synthetics computed using the Direct Solution Method, which allows us to take into account the correct phase shift of the PKiKP. We found that, in our region of study, the compressional velocity is slower in the uppermost inner core than the AK135 model. However, the sampled region is located in the Eastern hemisphere generally considered as faster and more attenuating than the AK135 model. This suggests significant regional departures from the simple hemispherical pattern reported in the literature.

We also deduce from the amplitude ratios that the attenuation is stronger than the global average at the top of the inner core in this region, as expected from its location in the eastern hemisphere. Our results confirm the presence of a low attenuation zone at the top of the inner core, followed by a highly attenuating layer centered around $35 \mathrm{~km}$ depth in the inner core, as found in the eastern hemisphere in previous studies. This zone is however thinner $(15 \mathrm{~km})$ and more attenuating, $\mathrm{Q}_{\alpha}$ reaching values less than 50 .
\end{abstract}

\section{Introduction}

The Inner Core Boundary (ICB) is a dynamic region which is the locus of the inner core growth as a result of iron crystallization induced by progressive earth cooling (e.g. Shimizu et al., 2005). As the inner core grows, light elements are expelled into the liquid outer core, driving chemical convection in the outer core. This is thought to be the main mechanism currently fueling the Earth's geodynamo (Jacobs, 1953; Loper, 1978; Loper and Roberts, 1981; Fearn et al., 1981).

The process of inner core growth is likely complex, and its further understanding relies on the ability of seismological studies to provide detailed information on several key parameters, among which: the density jump across the ICB, the P-velocity $\left(V_{p}\right)$ and its gradient, the bulk attenuation structure above and below the ICB, as well as the attenuation in shear below the ICB. A combination of body wave phases reflected, refracted or diffracted on the ICB have been used to constrain these parameters (Fig. 1).
It has been known for a long time that the $V_{p}$ gradient across the outer core presents a flattening in the last $\sim 150-200 \mathrm{~km}$ above the ICB. This region of the outer core was introduced by Bullen (1947) as the F layer to identify the negative velocity gradient detected by Jeffreys (1939), initially thought to produce the PKP precursors. Although these precursors were later attributed to the lowermost mantle (Cleary and Haddon, 1972; Doornbos and Husebye, 1972; Hedlin et al., 1997), the existence of a low-velocity gradient in the lowermost outer core has been confirmed in studies of core phases sensitive to this region (Souriau and Poupinet, 1991; Song and Helmberger, 1992).

The zone of reduced gradient is present in current reference seismological models based on body wave travel times, such as AK135 (Kennett et al., 1995). Geodynamically, this has been explained by a transition from neutral density stratification in the bulk of the outer core to a stable stratification at its base (Gubbins et al., 2008).

Many seismological studies have documented lateral variations in structure in the vicinity of the ICB, both at the base of the outer core

\footnotetext{
* Corresponding author.

E-mail address: aibourichene@live.fr (A. Ibourichene).
} 


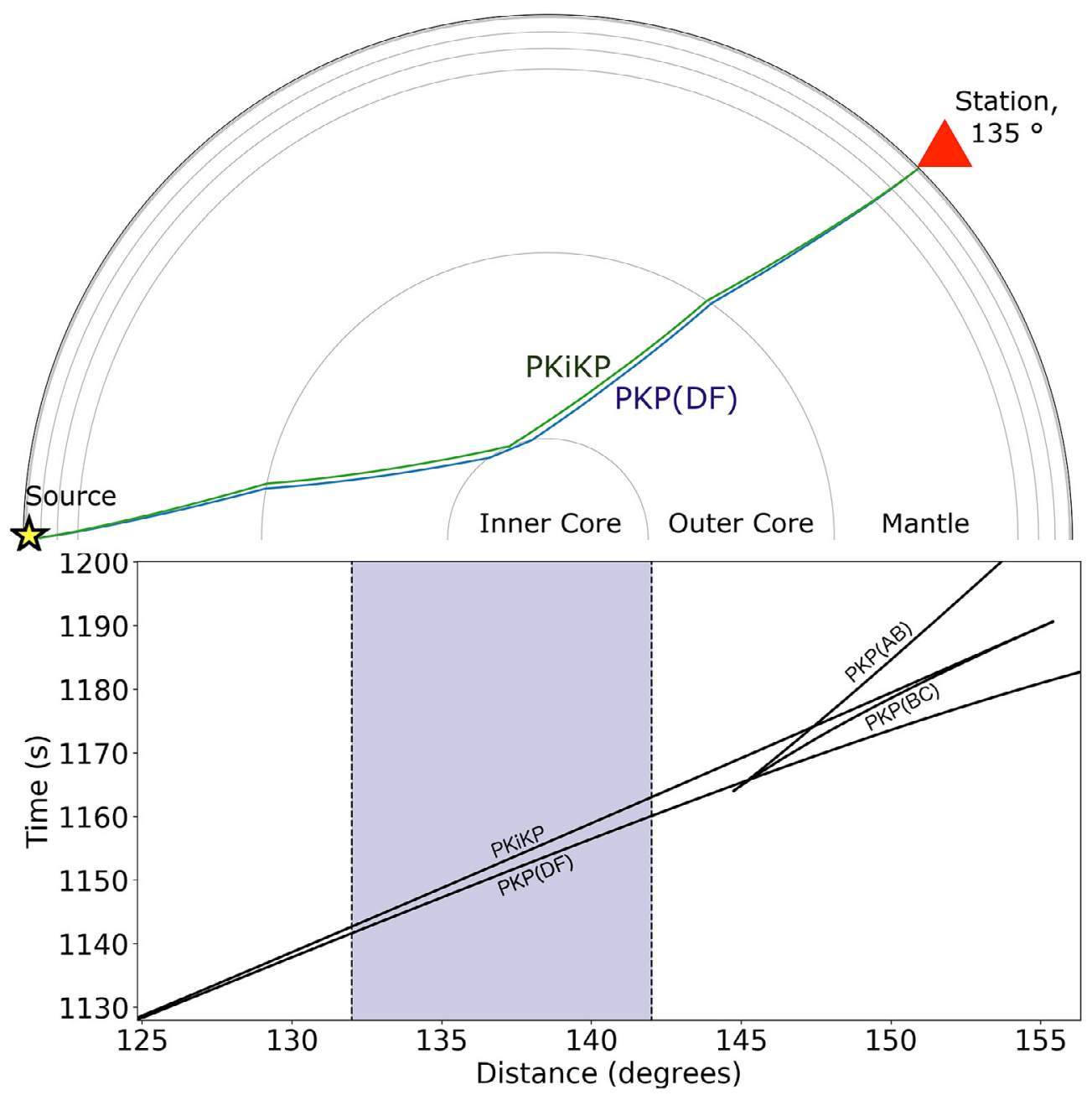

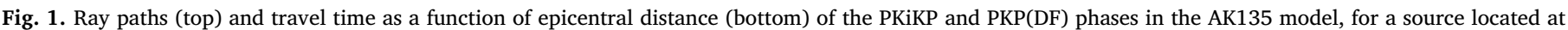

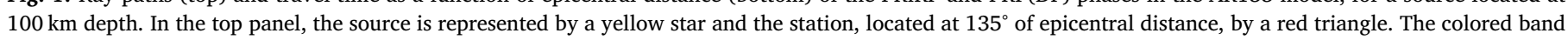
indicates the epicentral distance range of our study.

and at the top of the inner core. At the global scale, Tanaka and Hamaguchi (1997) first documented hemispherical differences in the top $500 \mathrm{~km}$ of the inner core, from measurements of differential $P K P_{B C}-P K P_{D F}$ travel times. More recent studies used differential measurements between the ICB reflected phase PKiKP at post-critical distances and the inner core refracted phase $P K P_{D F}$, the paths of which differ only in the immediate vicinity of the ICB, confirming faster velocities in the Eastern hemisphere than in the Western hemisphere (e.g. Garcia, 2002; Niu and Wen, 2001; Cao and Romanowicz, 2004; Yu, 2006; Waszek and Deuss, 2011; Ivan and He, 2017). Boundaries between hemispheres vary among different studies (e.g. Fig. 2).

Lateral variations in attenuation at the top of the inner core have also been found, with higher attenuation correlating with higher velocity (Cao and Romanowicz, 2004; Yu, 2006). This particular correlation between attenuation and velocity is the opposite of that found in the upper mantle. Proposed explanations include the presence of melt inclusions (Singh et al., 2000), the different size of crystals between the two hemispheres or variable concentration of impurities (Coté et al., 2008). The positive correlation could also arise from the alignment of anisotropic crystals, which could generate anisotropic scattering at the origin of energy loss (Cormier, 2007).

While the top $100 \mathrm{~km}$ of the inner core are thought to be isotropic, this hemispherical pattern extends deeper into the inner core, where it is manifested by differences in the strength of anisotropy, with weak anisotropy in the Eastern hemisphere and stronger anisotropy in the
Western hemisphere in the top 600-700 km of the inner core (Creager, 1999; Garcia and Souriau, 2000; Souriau, 2007; Sun and Song, 2008; Irving and Deuss, 2011).

Several models have been proposed to explain the hemispherical dichotomy. One of them (Alboussiere et al., 2012; Monnereau et al., 2010) implies a translation of the inner core due to thermal convection within the solid inner core. The shift from its equilibrium position would induce an asymmetry between a melting and a crystallising hemisphere. This model also suggests an explanation for the formation of the F layer, since enough melting and crystallisation could be produced on each side, to form a dense layer above the ICB, variably enriched or depleted in iron.

An alternative model (Sumita and Olson, 1999; Aubert et al., 2008; Gubbins et al., 2011) involves lateral variations in heat flow at the coremantle boundary (CMB), due to the presence of cold slabs sinking down to the $\mathrm{CMB}$. The temperature anomaly thus produced in the outer core would propagate down to the ICB to generate this degree-one pattern. However, neither of these models is able to explain the sharp hemisphere boundaries observed by seismologists (Geballe et al., 2013). More detailed regional studies comparing PKiKP and PKP(DF) waveforms have also found differences in structure within the same hemisphere (Stroujkova and Cormier, 2004; Attanayake et al., 2014).

While the depth extent of the F-layer and the velocity structure within it are not yet well constrained, there are also indications of lateral variations in correspondence with the hemispherical structure in 


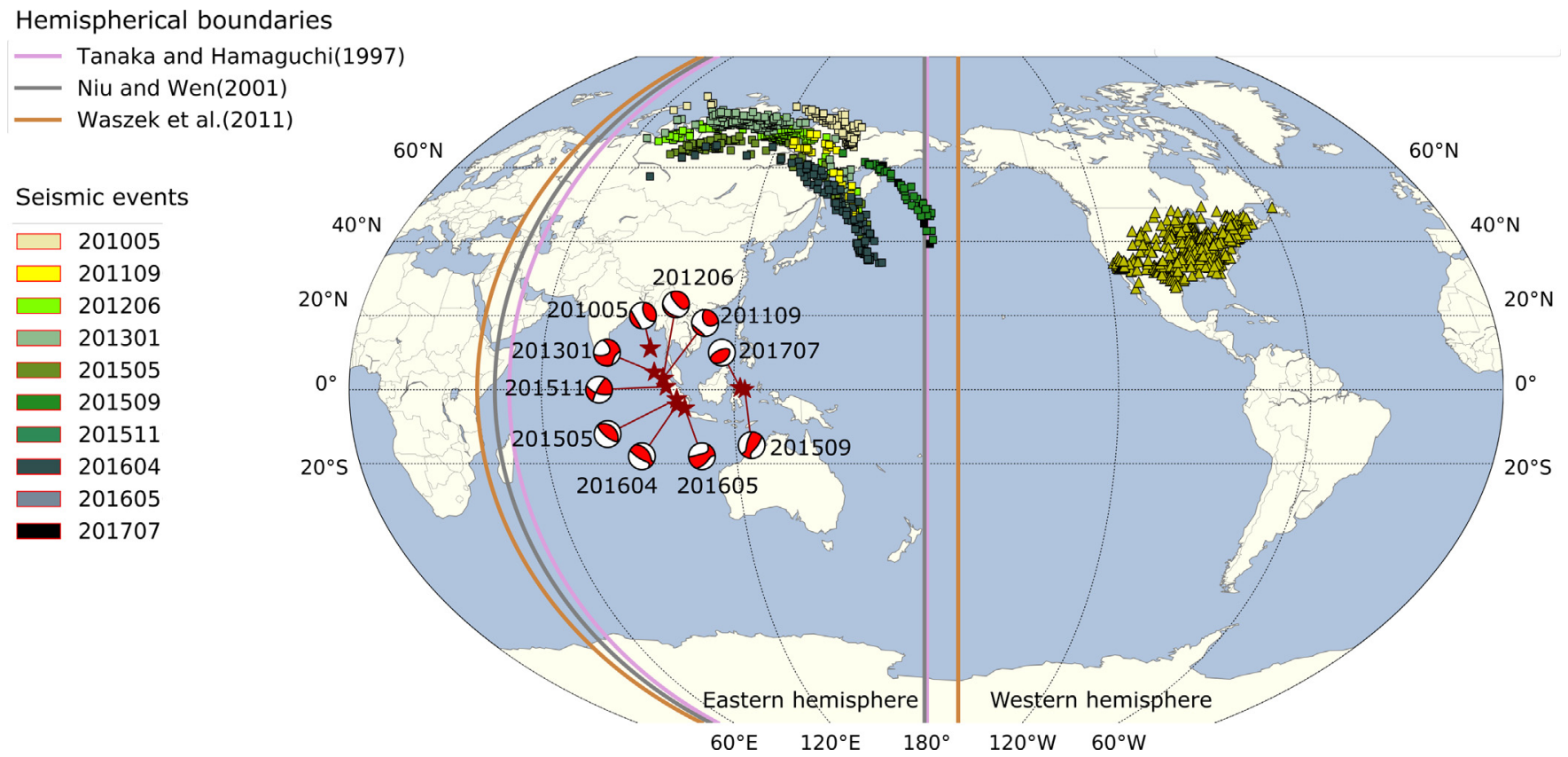

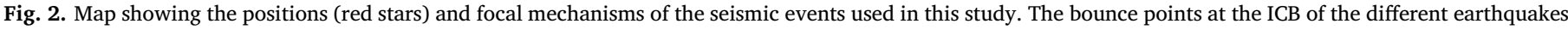

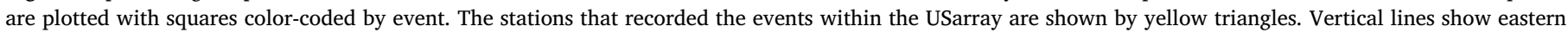
boundary of the Eastern hemisphere from several studies.

the inner core (Kaneshima et al., 1994; Yu et al., 2005; Souriau, 2015; Ohtaki and Kaneshima, 2015).

Finally, observations of amplitude anomalies in reflected PKiKP phases indicate the possible presence of short wavelength topography on the ICB (Cao et al., 2007; Zou et al., 2008; Dai et al., 2012; Tanaka and Tkalčić, 2015), with possible detection of temporal changes (Wen, 2006; Yao et al., 2015). Evidence for small scale scattering due to heterogeneities in the vicinity of the ICB have also been proposed from studies of the coda of core-sensitive phases (Vidale and Earle, 2000; Koper et al., 2004; Koper and Dombrovskaya, 2005; Cormier, 2007; Leyton and Koper, 2007; Adam and Romanowicz, 2015). Their presence was also confirmed by the broadening of the PKP(DF) with respect to $\mathrm{P}$ and short-scale variations in the residual times and amplitude ratios (Leyton and Koper, 2007; Cormier et al., 1998).

Here we describe the results of a regional study, aimed at documenting lateral variations of structure near the ICB at higher resolution. We considered ten earthquakes located around Sumatra and recorded in north America by the US Array (http://www.usarray.org) in the $132-142^{\circ}$ distance range, to investigate a region located beneath northeast Asia, using differential measurements of travel times and amplitude ratios of PKP(DF) and PKiKP. These two phases are well suited to investigate structure in the vicinity of the ICB, as their paths throughout the mantle and the outer core are very close, allowing us to eliminate many of the uncertainties related to heterogeneity in the traversed mantle. Using this pair of phases is challenging, because they are difficult to separate on the record in the distance range considered. We here present a new method to automate the identification of the PKP(DF) and PKiKP arrivals using the envelope of the signal.

In the next section, we describe the dataset and the method developed in this study. The following section presents the resulting measurements of differential travel times and amplitude ratios as well as their geographical patterns. Finally, we discuss the origin of these lateral variations and the relation between velocity and attenuation in the uppermost inner core.

\section{Data and method}

Because the body wave phases PKP(DF) and PKiKP travel along very similar paths except in the vicinity of the ICB, they have been widely used to study this region. However, they arrive closely in time (Fig. 1), so that their waveforms are difficult to separate on seismograms for the shorter distances. Current approches to address this rely on the crosscorrelation of the PKP(DF) and PKiKP waveforms (Waszek and Deuss, 2011; Cao and Romanowicz, 2004) or on manual picking of the maximum of each phase (Niu and Wen, 2001; Niu and Wen, 2002; Wen and Niu, 2002; Yu, 2006; Yu, 2006; Ohtaki et al., 2012; Ohtaki and Kaneshima, 2015).

Some authors have also developed waveform modeling approaches (Cormier et al., 2011; Attanayake et al., 2014; Garcia, 2002).

Here we restrict our data collection to the $132-142^{\circ}$ epicentral distance range in which the PKiKP-PKP(DF) differential time exceeds 1s. The upper bound is set at $142^{\circ}$ to avoid contamination from the PKP (AB) and PKP(BC) phases (Fig. 1). In this distance range, the PKP(DF) waves samples the top $100 \mathrm{~km}$ of the inner core beneath Eastern Russia, which is in the faster and more attenuating Eastern hemisphere (Fig. 2).

We collected and processed waveforms for 10 earthquakes of $5.5<M w<7$ and depth greater than $30 \mathrm{~km}$ located in the Sumatra region, Indonesia (Table 1), and recorded on the USArray broadband stations ( http://www.usarray.org). Such a large and dense array provides an opportunity to investigate small-scale heterogeneities near the ICB. We limited the range of magnitude to avoid source complexity and inspected records to only keep those events for which a sufficient number of simple PKP(DF) and PKiKP waveforms were found. Source parameters presented in Table 1 are those provided in the Harvard CMT catalog (http://globalcmt.org).

The frequency content of the PKiKP and PKP(DF) phases is concentrated around $1 \mathrm{~Hz}$. A $0.4-1.8 \mathrm{~Hz}$ filter is therefore applied before a visual check to make sure the PKP(DF) arrival is not masked by precursors which are abundant in the distance range considered (e.g. Cleary and Haddon, 1972; Doornbos and Husebye, 1972; Hedlin et al., 1997; Margerin and Nolet, 2003; Mancinelli and Shearer, 2013). Those PKP(DF) data that are polluted by precursors are removed from our database. Records are also corrected for instrument response and converted to velocity.

Because of their close arrival times, the PKiKP and PKP(DF) phases often require manual picking (Niu and Wen, 2001; Niu and Wen, 2002; 
Table 1

List of the seismic events and hypocentral coordinates according to the Harvard CMT/global CMT catalog considered in this study.

\begin{tabular}{|c|c|c|c|c|c|c|}
\hline Event & Date & Origin Time (UT) & Latitude, ${ }^{\circ} \mathrm{N}$ & Longitude, ${ }^{\circ} \mathrm{E}$ & Depth, km & Magnitude \\
\hline 201005 & 2010.05 .31 & $19: 51: 49.9$ & 11.16 & 93.70 & 127.9 & 6.5 \\
\hline 201109 & 2011.09.05 & $17: 55: 14.8$ & 2.88 & 97.86 & 94.6 & 6.7 \\
\hline 201206 & 2012.06 .23 & $4: 34: 54.9$ & 2.98 & 97.77 & 104.5 & 6.1 \\
\hline 201301 & 2013.01 .10 & 13:47: 6.0 & 4.54 & 95.03 & 45.2 & 5.7 \\
\hline 201505 & 2015.05 .15 & $20: 26: 58.3$ & -2.61 & 102.14 & 158.4 & 6.0 \\
\hline 201509 & 2015.09 .13 & 1:13:14.2 & 0.12 & 123.58 & 146.5 & 5.5 \\
\hline 201511 & 2015.11 .08 & 9:34:59.0 & 0.78 & 98.78 & 87.4 & 5.7 \\
\hline 201604 & 2016.04 .10 & $2: 14: 37.8$ & -4.42 & 102.05 & 48.5 & 5.7 \\
\hline 201605 & 2016.05 .02 & $4: 21: 25.2$ & -5.26 & 104.49 & 125.4 & 5.7 \\
\hline 201707 & 2017.07.15 & $12: 12: 22.5$ & 0.44 & 121.95 & 125.8 & 5.9 \\
\hline
\end{tabular}

Wen and Niu, 2002; Yu, 2006; Yu, 2006; Ohtaki et al., 2012; Ohtaki and Kaneshima, 2015) or visual checking (Waszek and Deuss, 2011; Cao and Romanowicz, 2004) to be distinguished. A new method relying on the envelope of the seismic signal and its first and second derivatives is developed here to automate the detection of these phases, as follows.

The envelope of a seismic signal $\mathrm{u}(\mathrm{t})$ is defined as:

$\operatorname{env}(t)=\sqrt{u(t)^{2}+H[u(t)]^{2}}$

where $\mathrm{H}(\mathrm{u}(\mathrm{t}))$ is its Hilbert transform.

The envelope is commonly used in seismology to monitor volcanic activity (De Siena et al., 2013), measure the seismic energy released by rockfalls (Hibert et al., 2011), infer the parameters of a seismic source (Mayeda and Walter, 1996) or describe the scattering effect in the coda of a seismic station $($ Sato, 1984).

This tool is here extended to the detection of the PKP(DF) and PKiKP waveforms. A phase arrival is indicated by a sharp increase in the envelope (Fig. 3) which can be precisely located through its derivatives. Indeed, its first derivative contains information on the envelope tendency, i.e. whether the envelope increases or decreases with time, whereas impulses in the second derivative emphasize changes in the envelope behavior. The PKP(DF) and PKiKP arrivals are thus identified by two conditions applied on the envelope derivatives and one on the seismic signal:

- (i) a positive first derivative that indicates an increase in the envelope and

- (ii) a maximum in the second derivative that implies a sharp onset in the envelope and

- (iii) PKP(DF) and PKiKP have opposite polarity in the seismic signal as expected.

The conditions (i), (ii) and (iii) are jointly used to identify the two phases. In the following, the theoretical travel times predicted by TauP (Crotwell et al., 1999) in model $A K 135$ (Kennett et al., 1995) are labelled $T_{A K 135}^{P K P(D F)}$ and $T_{A K 135}^{P K i K P}$ for PKP(DF) and PKiKP, respectively.

Since the PKP(DF) is predicted to arrive before the PKiKP, we first concentrate on its arrival. We start by searching for an increase in signal-to-noise ratio $\pm 5 \mathrm{~s}$ around the predicted $T_{A K 135}^{P K P(D F)}$ to allow errors in the source parameters and make a preliminary detection of the PKP (DF) arrival.

To refine this arrival time, we then consider the (i) and (ii) conditions. The time windows on which the (i) condition is satisfied are filled in red in the first derivative plot presented in Fig. 3(a) whereas the (ii) condition imposed on the second derivative of the envelope is highlighted by a maximum, also filled in red in Fig. 3(a). The time at which both of these conditions are fulfilled are finally identified as the PKP (DF) arrival time, hereafter labelled $T_{\text {data }}^{P K P(D F)}$ for the data and $T_{\text {synth }}^{P K P(D F)}$ for the synthetics.

We then look for the PKiKP arrival around the arrival time expected after the determination of the PKP(DF) arrival, i.e. $T_{0}^{P K i K P}=T^{P K P(D F)}+\left(T_{A K 135}^{P K i K P}-T_{A K 135}^{P K P(D F)}\right)$, where $T^{P K P(D F)}$ refers to $T_{\text {data }}^{P K P(D F)}$ or $T_{\text {synth }}^{P K P(D F)}$. This phase is also identified by the (i) and (ii) conditions and the expectation that the PKP(DF) and PKiKP waveforms have opposite polarity in the seismic signal, i.e. the (iii) condition. This last condition is essential to pick the PKiKP onset time correctly. Fig. 3(a) compares the PKiKP arrival times before (green dashed line, D2-PKiKP) and after (red dashed line) applying this condition on the waveforms polarity. This arrival time used in the next sections takes into account the requirement on the PKiKP-PKP(DF) inverse polarity.

The search of the PKiKP starts $0.7 \mathrm{~s}$ after the PKP(DF) arrival to allow any reasonable departure from the reference $1 \mathrm{D}$ model. This time window ensures that the PKiKP does not interfere with the PKP(DF) waveform. At the lowest epicentral distance considered, i.e. $132^{\circ}$, the PKiKP is detected if arriving no later than $0.3 \mathrm{~s}$ before its predicted arrival time. As the epicentral distance and thus the PKiKP-PKP(DF) differential travel time increase, the PKiKP search starts earlier, allowing for stronger departure from the 1D model. For instance, at $133^{\circ}$, the search window begins $0.5 \mathrm{~s}$ before the PKiKP arrival predicted by Taup.

An example of the picking procedure is given in Fig. 3, which shows a vertical component velocity record, its envelope and the first and second derivatives of the envelope as well as the relationship of the second derivative of the envelope with the signal itself, its envelope, and the first derivative of the envelope, illustrating the power of using the derivatives of the envelope.

Unlike methods relying on cross-correlation, our approach is free of any assumptions on the precise phase difference between PKP(DF) and PKiKP. It also does not require any corrections for possible broadening of the two phases due to differential attenuation.

The main purpose of the procedure described in the previous section is the identification of the PKiKP arrival, so that the portions of record containing the PKP(DF) and PKiKP waveforms can be separated for subsequent differential measurements of travel times and amplitudes. This is illustrated in Figs. 4 and 5, which show synthetic and observed record sections, respectively, of the seismic signals and their envelopes, aligned on the PKiKP arrival time determined by our method. In the data plot (Fig. 5), the picked PKP(DF) arrival time oscillates due to an interplay between the rate of increase of the envelope at the PKP(DF) onset and the noise level. A PKP(DF) signal stronger than the noise exhibits a sharp envelope increase and provides a precise arrival time for this phase. The presence of precursors smoothes the envelope trend, resulting in a less precise pick of PKP(DF). However, this pick is not used further, as it is only considered for determining the time window in which the PKiKP arrival is sought.

\section{Differential travel times and amplitude ratios}

In the previous section, we described the method which allows us to separate time windows corresponding to the arrivals of PKP(DF) and PKiKP. Here we proceed to describe the measurement of differential travel times and amplitude ratios. 
a

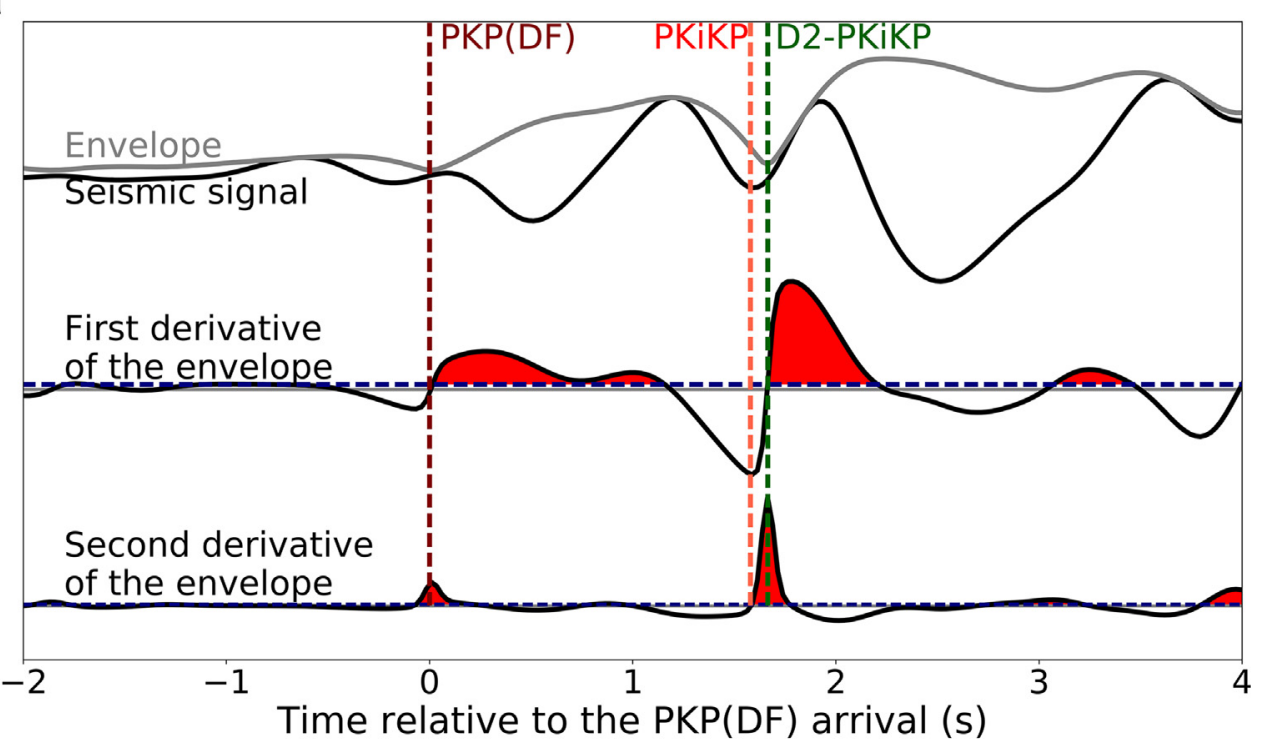

b

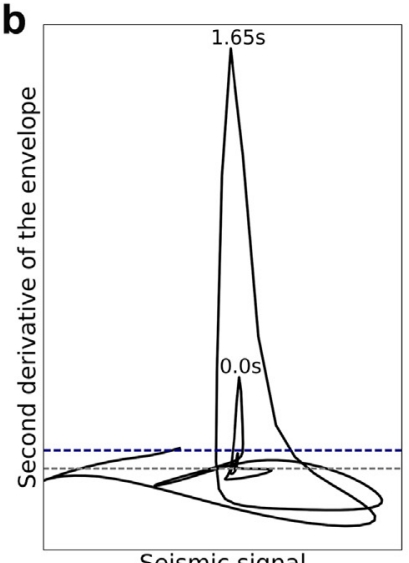

Seismic signal

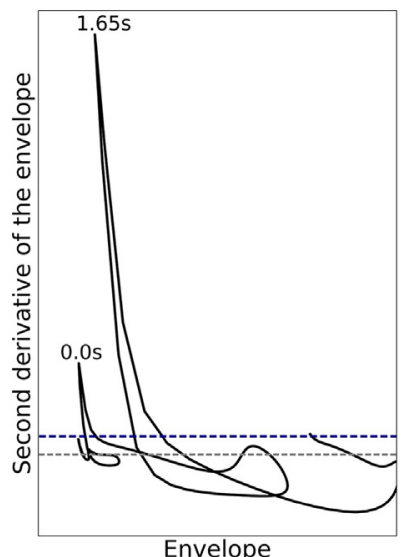

Envelope

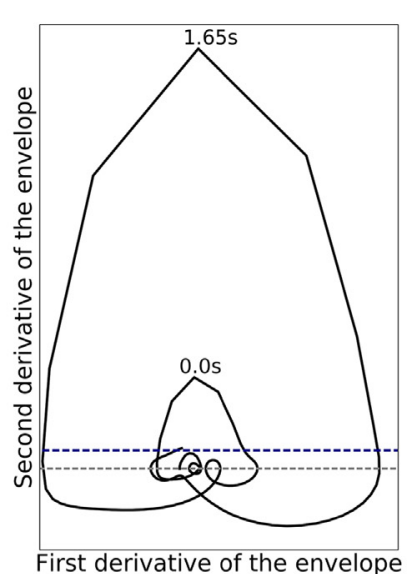

First derivative of the envelope
Fig. 3. a. Example of seismic velocity record, its envelope and first and second derivative of the envelope. The record is for event 201206 (Table 1) observed at station KSPA (distance: $135.06^{\circ}$ ). The zero time corresponds to the PKP(DF) arrival time obtained with our method and is highlighted by the dashed brown line. The vertical orange dashed line corresponds to the PKiKP arrival time obtained with our method whereas the green dashed line (D2PKiKP) refers to the arrival time that would be obtained if the condition of opposite polarity in the PKP(DF) and PKiKP waveforms were not taken into account. The part of the signal exceeding the ambient noise and in which the PKP(DF) and PKiKP are searched for is indicated in red. The grey line is the reference zero line, and the dashed blue line corresponds to the noise level in the first or second derivative of the envelope. This noise level is defined as the averaged abolute signal recorded between 5 and $2 \mathrm{~s}$ before the PKP(DF). b. Second derivative of the envelope as a function of the seismic signal (left), envelope(middle) and first derivative of the envelope (right). The maxima in these plots correspond to the PKP(DF) and PKiKP arrivals identified at $0 \mathrm{~s}$ and 1.65 $\mathrm{s}$ respectively in a. The dashed blue line corresponds to the estimated background noise level in the second derivative and the grey line to the reference zero line.

\subsection{Differential travel times}

In previous work, such measurements often relied on the crosscorrelation of the waveforms of the two phases, assuming that PKP(DF) and PKiKP waveforms have similar shapes but opposite polarity (e.g. McSweeney et al., 1997; Creager, 1999; Engdahl et al., 1974; Koper et al., 2003; Waszek and Deuss, 2011).

However, we note that the phase shift between these PKP(DF) and PKiKP phases increases with epicentral distance between 132 and $142^{\circ}$ (e.g. Fig. S1). Cross-correlating the PKP(DF) and PKiKP waveforms could therefore produce an error as a function of the distance in the residual differential travel times.

To avoid this problem, instead of directly comparing the waveforms of the two phases in the observed records, we first compute synthetic waveforms for each event in model AK135, using the Direct Solution Method which takes into account the focal mechanism of the seismic event (DSM, Cummins et al., 1994; Geller and Takeuchi, 1995) and filter them in the same frequency band as the data. The PKP(DF) and PKiKP arrivals for the data and synthetics are then determined using the envelope and its derivatives and labelled $T_{\text {data }}^{P K P(D F)}, T_{\text {data }}^{P K i K P}, T_{\text {synth }}^{P K P(D F)}$ and $T_{\text {synth }}^{\text {PKiKP. }}$.

We then cross-correlate the observed waveform with the corresponding synthetic waveform, for each of the two phases. For PKP(DF), the time window used for this cross-correlation is $1.2 \mathrm{~s}$ except in the case where the PKiKP-PKP(DF) differential travel time is lower than this duration, which is observed at low epicentral distance. In such a case, the window duration is reduced to the PKiKP-PKP(DF) differential travel time. For PKiKP, the same 1.2s window length is kept for all the records. The cross-correlation eliminates the error in the PKP(DF) arrival time.

For each phase, the synthetic and observed waveforms are allowed to shift by less than $0.6 \mathrm{~s}$ in order to detect any departure from the reference model. Larger time shifts indicate a misidentification of the PKiKP or PKP(DF) phases either in the synthetics or the data.

We then obtain absolute PKP(DF) and absolute PKiKP travel time residuals defined as:

$\delta t^{P K P(D F)}=T_{\text {data }}^{P K P(D F)}-T_{\text {synth }}^{P K P(D F)}$

$\delta t^{\text {PKiKP }}=T_{\text {data }}^{\text {PKiKP }}-T_{\text {synth }}^{\text {PKiKP }}$

The differential travel time residual is computed by taking the difference between these absolute residuals:

$\delta T^{I C B}=\delta t^{P K i K P}-\delta t^{P K P(D F)}=\left(T^{P K i K P}-T^{P K P(D F)}\right)_{\text {data }}-\left(T^{P K i K P}-T^{P K P(D F)}\right)_{\text {synth }}$

\subsection{Amplitude ratios}

Similarly, we used the PKP(DF) and PKiKP arrival times determined earlier to compute the peak-to-peak amplitude ratios between synthetics and data for each phase. These arrival times mark the onset of the PKP(DF) and PKiKP waveforms in which the peak-to-peak 

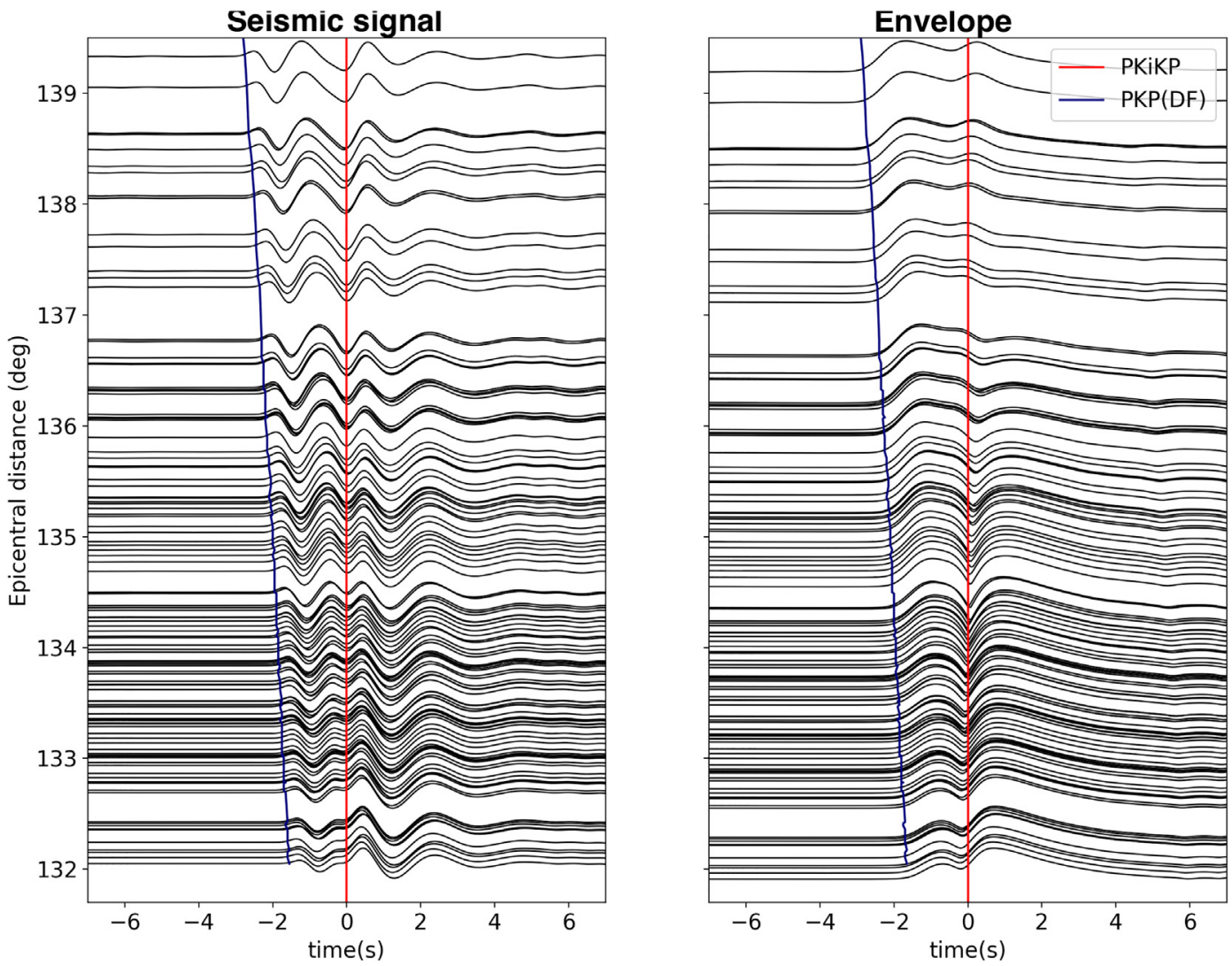

Fig. 4. Record section of synthetics (left) and their envelopes (right) aligned on the PKiKP arrival time determined by our method. The synthetics have been computed for event 201005 (Table 1). The red and blue lines correspond respectively to the PKiKP and PKP(DF) arrivals identified with our method.
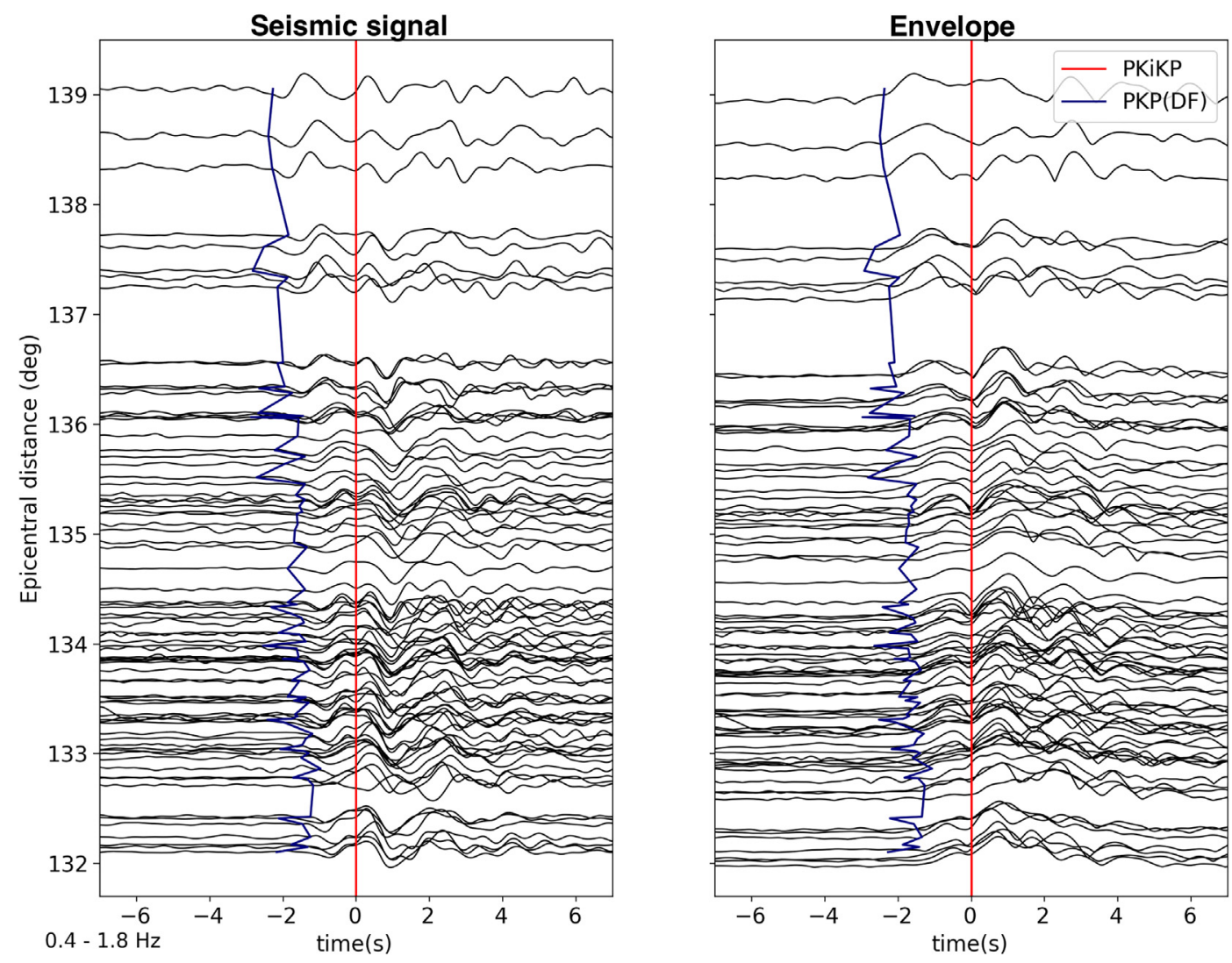

Fig. 5. Same as Fig. 4 for the real data corresponding to the same event. 
amplitude of each phase is measured with the extrema satisfying the following conditions:

- (j) the PKP(DF) and PKiKP waveforms have an opposite polarity in both data and synthetics and

- (jj) the data and synthetics present similar waveform shapes for each phase.

That is, we check that, for example, if the first peak of the PKP(DF) is a maximum in the synthetics, the corresponding first peak in the PKiKP waveform is a minimum. On the other hand, the data are required to present the same behavior, i.e. the PKP(DF) starts by a maximum and the PKiKP by a minimum. These polarities have to be similar for all the records of the same event.

Applying these conditions to the PKP(DF) and PKiKP waveforms allows us to retrieve the phase amplitude ratio for the PKiKP and the PKP(DF), between the data and synthetics:

$A^{P K i K P}=\frac{A_{\text {data }}^{\text {PKiKP }}}{A_{\text {synth }}^{\text {PKiKP }}}$

$A^{P K P(D F)}=\frac{A_{\text {data }}^{P K P(D F)}}{A_{\text {synth }}^{P K P(D)}}$

The relative amplitude ratios are then evaluated as:

$A^{I C B}=\frac{A^{P K i K P}}{A^{P K P(D F)}}=\frac{A_{\text {data }}^{\text {PKIKP }}}{A_{\text {data }}^{\text {PKP }(D F)}} * \frac{A_{\text {synth }}^{P K P(D F)}}{A_{\text {synth }}^{\text {PKiKP }}}$

These amplitude ratios and travel time residuals, initially determined automatically, are later visually inspected to finally obtain 627 residual differential travel times and 538 amplitude ratios.

\section{Results}

We first present the results derived from the residual travel times and then concentrate on the amplitude ratios.

\subsection{Differential travel time residuals}

In this study, we cross-correlate observed and synthetic waveforms calculated for model AK135 in order to evaluate the residual travel times.

The right panel of Fig. 6 presents the residual travel times measured with our method (black squares). Negative residual travel times suggest that the uppermost inner core is slower than in the AK135 model. Since these results disagree with the well-documented global hemispherical pattern, we compared our results with papers measuring the PKiKP-PKP (DF) residual travel times in our study area.

Ivan et al. (2018) calculated the PKiKP-PKP(DF) residual differential arrival times with respect to the AK135 model and reported slightly negative values in Eastern Russia, which agree with our results. Our measurements also agree with $\mathrm{Yu}$ et al. (2017), who analysed the hemispheric boundary around $180^{\circ}$ and found PKiKP-PKP(DF) residual differential travel times fluctuating around zero beneath Eastern Russia. Recently, Godwin et al., 2018 proposed a velocity model beneath the North Pacific in which Vp is lower than in AK135 beneath $16.3 \mathrm{~km}$ in the inner core. Such a model could explain our observations.

The regional average in differential travel time residuals is presented in the form of a cumulative histogram binned in $0.1 \mathrm{~s}$ steps between -0.6 and $0.6 \mathrm{~s}$, the measurement error is estimated to be less than 0.05 s (Fig. 7). Most of our measurements are comprised in a narrow range extending from -0.4 to 0 s with an approximately gaussian distribution. If interpreted in terms of structure at the top of the inner core, negative residuals describe a structure that is slower than AK135 in this part of the Eastern Hemisphere.

In order to investigate shorter wavelength variations in the vicinity of the ICB, we present the travel time residuals in map view, plotted at the bounce point location of the PKiKP on the ICB (Fig. 8). At this latitude and radius of the ICB, $1^{\circ}$ roughly corresponds to $10-15 \mathrm{~km}$. The map of the differential travel time residuals exhibits patches that are coherent over distances on the order of $100 \mathrm{~km}$.

\subsection{Amplitude ratios}

We now consider the amplitude ratios in the same way as for the differential travel time residuals. The cumulative histogram of the amplitude ratios (Fig. 9a) is broader and less Gaussian than in the case of the travel time residuals, spanning from 0.1 to 1.9 , with a mean around 1.3. Interpreted in terms of attenuation at the top of the inner core, this indicates a more attenuating structure than in the AK135 model, which agrees with the characteristics of the Eastern hemisphere.

Expressed in terms of properties at the top of the inner core, the distribution of amplitude ratios and travel time residuals, on average, do not support the positive correlation between the compressional velocity and attenuation found at the global scale (Tanaka and Hamaguchi, 1997; Cao and Romanowicz, 2004).

We further investigate the evolution of the amplitude ratios as a function of epicentral distance in Fig. 10b. We note a regular increase in the relative amplitude ratios between 132 and $136^{\circ}$ whereas the data are more dispersed at larger epicentral distances (Fig. 10b). The increase in the amplitude ratios between 132 and $136^{\circ}$ suggests that this parameter may be very sensitive to the attenuation profile within the uppermost the inner core. This trend is not observed at greater epicentral distance, which limits it to the uppermost $50 \mathrm{~km}$ of the inner core.

The 1D model entered in DSM to compute synthetics assumes that $Q_{\kappa}$ and $Q_{\mu}$ are fixed at 628 and 85.03 respectively within the inner core, corresponding to $Q_{\alpha}=339$. $Q_{\alpha}$ can be calculated with the following expression:

$\frac{A_{P K P(D F)}}{A_{P K i K P}}=\exp ^{\frac{-\omega t^{*}}{2}}$

replace « exp » by «e »

replace $\ll Q \alpha$ can be calculated with the following expression: » by «In order to find the attenuation structure within the uppermost inner core explaining our amplitude ratios, (8)e inverted $A_{P K P}(D F)$ and $A_{P K i K P}$ the peak-to-peak amplitude of the PKP(DF) and PKiKP phases respectively, $\omega$ the angular velocity and $f$ the frequency so that $\mathrm{f}=1 \mathrm{~Hz}$ and

$t^{*}=\int \frac{d s}{Q_{\alpha} v}$

where ds (space missing)

whereds refers to the PKP(DF) ray path within the inner core, $Q_{\alpha}$ the quality factor and $v$ the compressional velocity.

To investigate the attenuation of the uppermost inner core, we invert for the $Q_{\alpha}$ structure in the inner core after dividing the uppermost $100 \mathrm{~km}$ of the inner core into 3 or 4 layers of varying thicknesses.

For each model, the residual is calculated and we present here the model having the lowest residual (shown in red in Fig. 10a), that corresponds to a 3 layers model with boundaries close to those proposed by Waszek and Deuss (2013) (blue lines in Fig. 10a). At depths greater than $106 \mathrm{~km}$ below the ICB, we fix the model to the value entered in DSM $\left(Q_{\alpha}=339\right.$, shown in black in Fig. 10a).

We find that the uppermost $27.5 \mathrm{~km}$ of the inner core is weakly attenuating with $Q_{\alpha} \sim 453$. The transition between the non-attenuating outer core and the inner core could partly explain this large value of $Q_{\alpha}$, especially if $Q_{\alpha}$ decreases slowly between the liquid and solid core instead of abruptly jumping as imposed by the parametrization in our model. Between 27.5 and $42.5 \mathrm{~km}$ below the ICB, we find that $Q_{\alpha}$ is particularly low, around 30 and then increases to $\sim 420$ at greater depths. The strongly attenuating layer is similar to that of Waszek and Deuss (2013) although somewhat thinner ( $15 \mathrm{~km}$ instead of $27.5 \mathrm{~km})$ and more attenuating. Cao and Romanowicz (2004) proposed a smooth increase from $Q_{\alpha}=100-200$ between 32 and $85 \mathrm{~km}$ beneath the ICB in the Eastern hemisphere. Our results indicate that the region of high attenuation, at least in this part of the eastern hemisphere, may be 

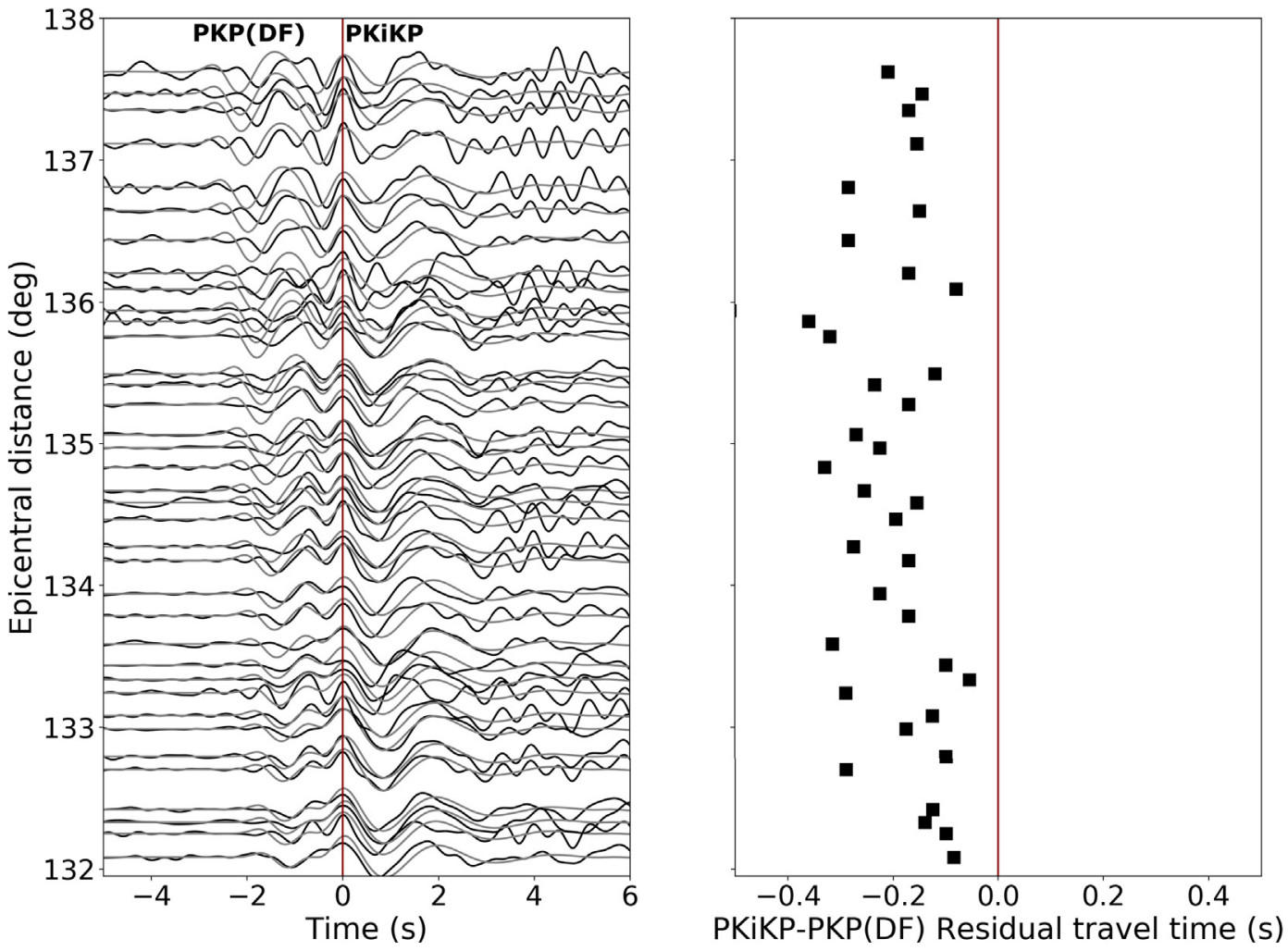

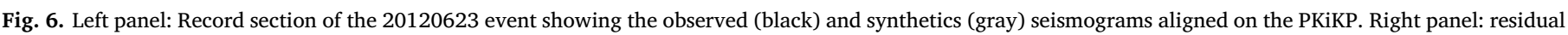
PKiKP-PKP(DF) differential travel times measured for the traces shown on the left panel.

thinner and more attenuating, suggesting the presence of mush (e.g. Cormier and Li, 2011; Roberts et al., 2003; Cao and Romanowicz, 2004) or slurry (e.g. Shimizu et al., 2005; Zou et al., 2008). However, the actual boundaries between high and low attenuation layers may not be sharp and the low $Q_{\alpha}$ values somewhat less extreme. A more comprehensive sampling of model space to investigate this further is beyond the scope of this paper.

Our model has a residual variance of about 0.58 (compared to 0.72 for the Waszek and Deuss (2013) model and fits the trend in the data better (Fig. 10b), especially in the distance range 134-139 $)$. The comparison between our measured relative amplitude ratios and the ones predicted by our model and that of Waszek and Deuss (2013) is

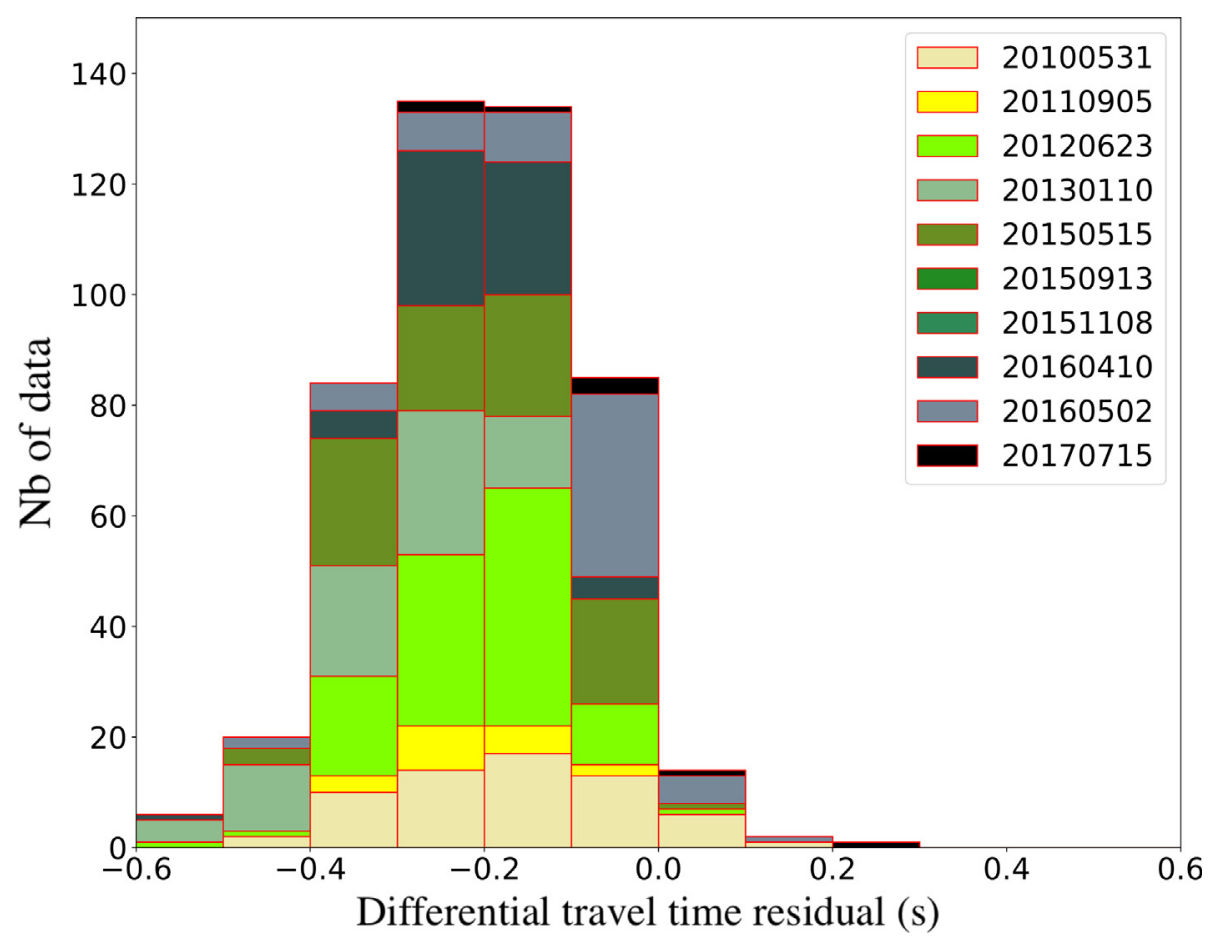

Fig. 7. Cumulative histogram of the PKiKP-PKP(DF) residual differential travel times for all the seismic events. 


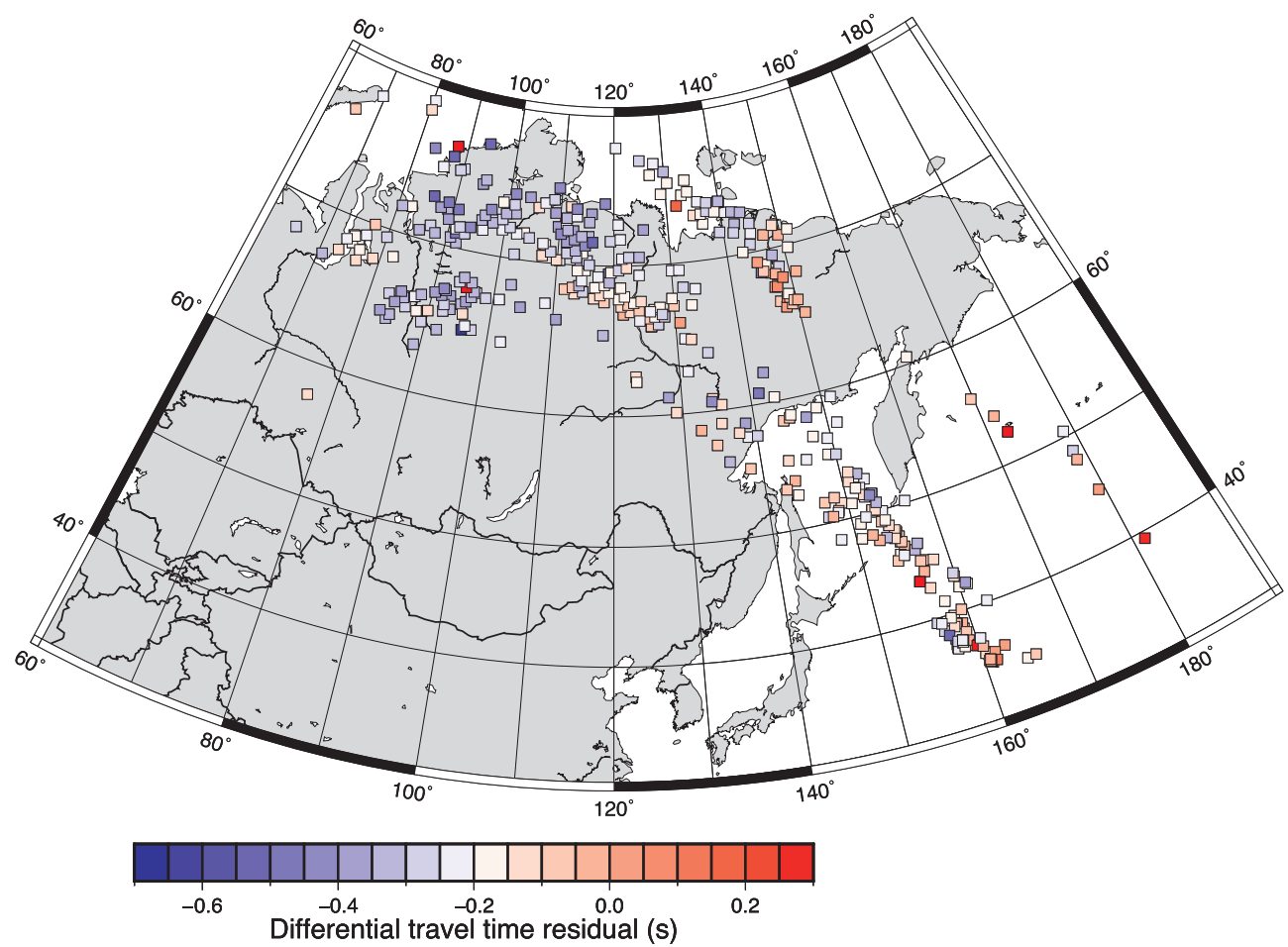

Fig. 8. Map of the PKiKP-PKIKP differential travel time residuals for the seismic events considered. Residuals with respect to AK135 are plotted at the reflection point of the PKiKP phase and color-coded by value.

a

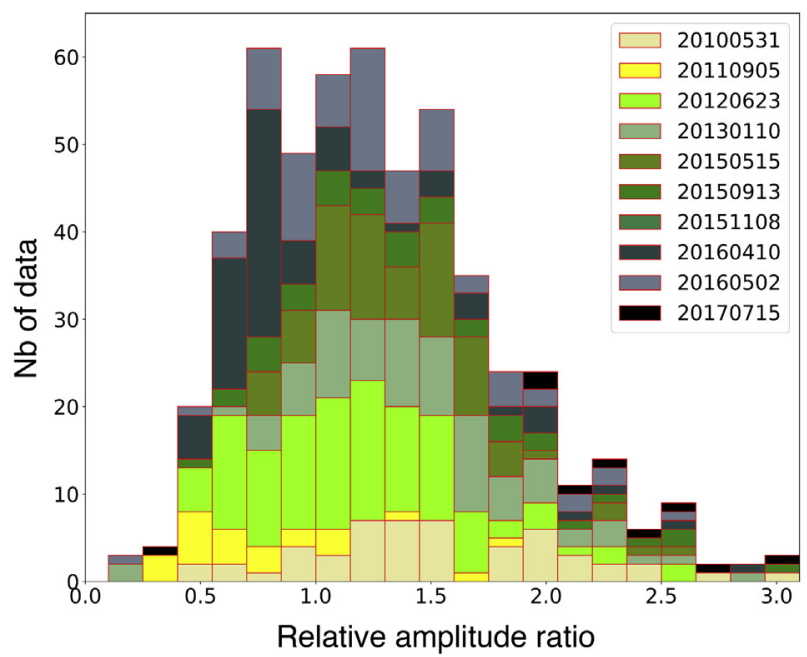

b

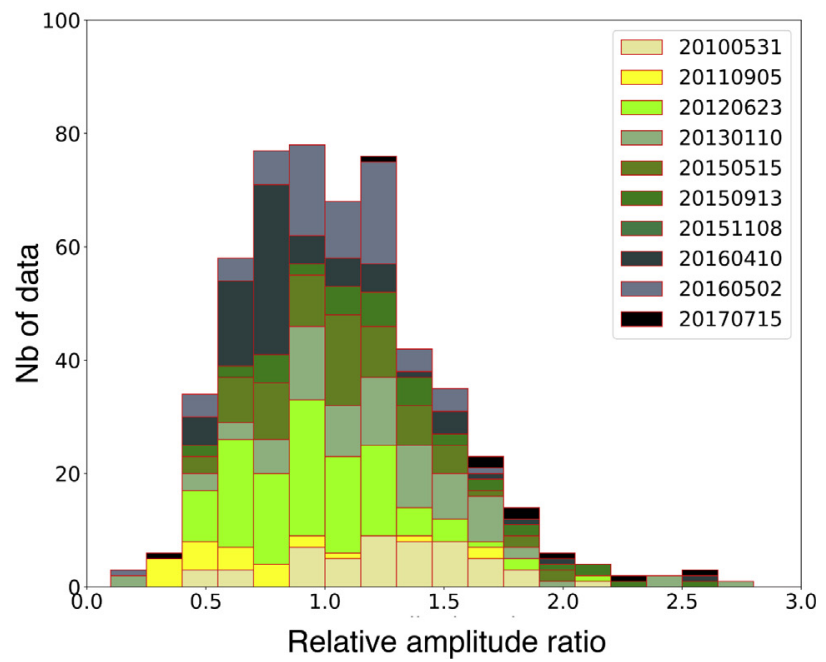

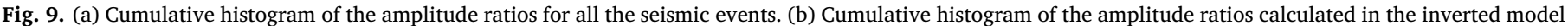
for all the seismic events (e.g. Fig. 10a).

presented in Fig. 10b. In particular, the increase between 132 and 136 degrees we previously discussed is correctly predicted as well as the slow decrease observed at greater epicentral distances.

Fig. 9b presents the cumulative histogram of the $A^{I C B}$ amplitude ratios once corrected for the $Q_{\alpha}$ structure inverted. The cumulative histogram of the corrected amplitude ratios (Fig. 9b) presents a tighter distribution around a value of 1 , that is also closer to a Gaussian.

The lateral variations in amplitude ratios, after correction for the 1D trend, are presented in map view in Fig. 11. There is no particular pattern in the futuctuations, which can vary by a factor of two over length scales of $\sim 10 \mathrm{~km}$ across the entire region sampled. These fuuctuations suggest either a rough ICB with possible topography or fluctuatulons
heterogeneities at very short wavelengths within the inner core.

\section{Relation between amplitude ratios and differential travel time residuals}

In order to further analyze the relation between velocity and amplitude at the regional scale, the amplitude ratios are plotted with respect to the travel time residuals for events 201206 and 201301 in Fig. 12.

The amplitude ratios and travel time residuals are not correlated for the two events. The velocity/attenuation relation does not seem to be valid at regional scale. 


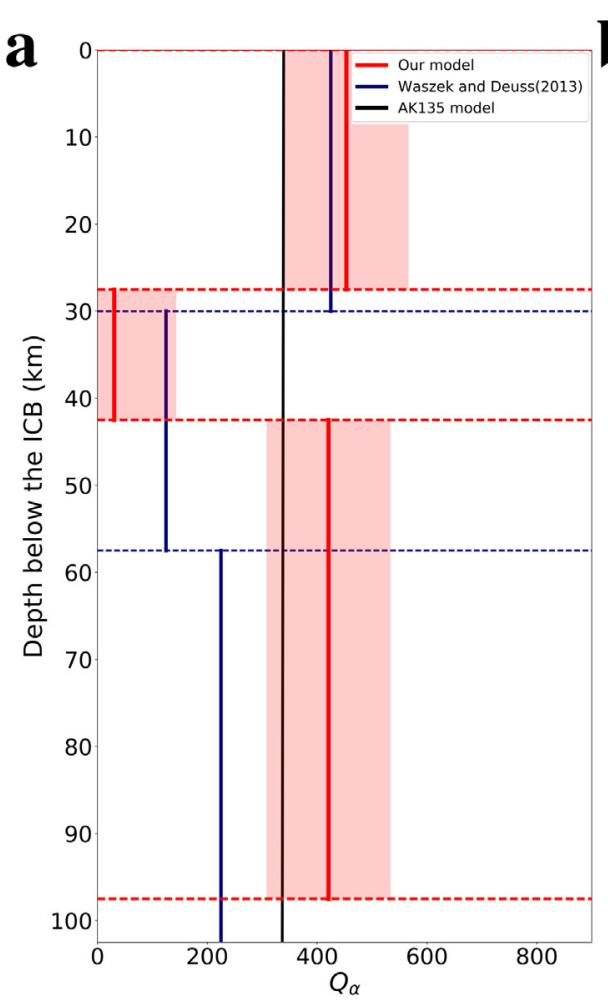

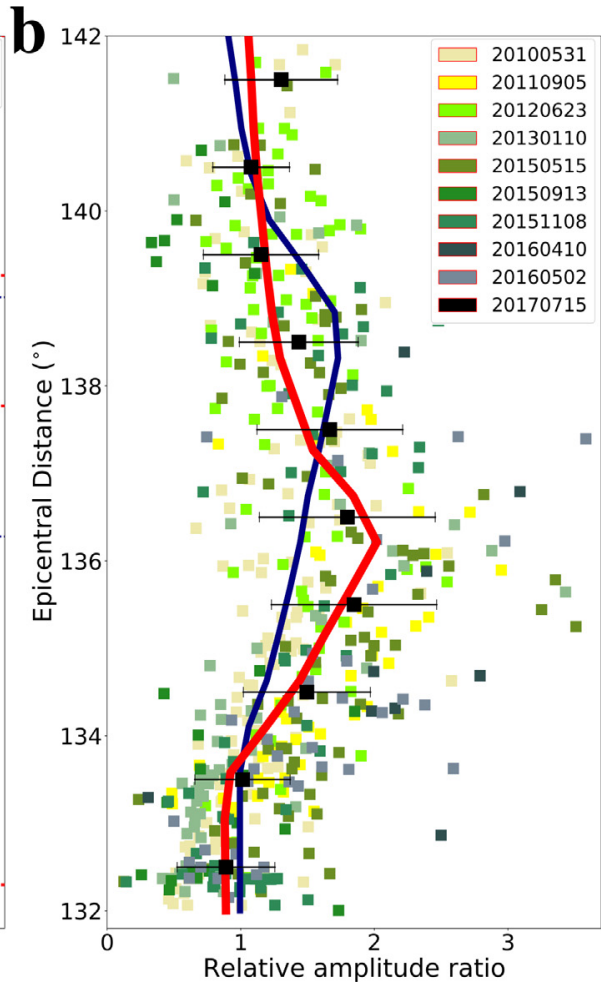

Fig. 10. (a) Evolution of $\mathrm{Q}_{\alpha}$ with depth in the uppermost inner core. The model inverted from the relative amplitude ratio is presented in red and the light red bands represent the error on this model. The $\mathrm{Q}_{\alpha}$ quality factor predicted by the reference 1D model used to compute the relative amplitude ratios is shown in black and the model proposed by Waszek and Deuss (2013) is indicated in blue. (b) Relative amplitude ratios as a function of the epicentral distance (color-coded by seismic event). The black squares refer to the mean of the relative amplitude ratios over one degree of epicentral distance and the standard deviations are indicated by the black lines. The relative amplitude ratios calculated in the inverted model are shown in red and those predicted in the model of Waszek and Deuss (2013) are shown in blue.

\section{Origin of our observation}

The variations observed in the travel time residuals or amplitude ratio maps could either originate from the uppermost inner core (i.e. PKP(DF)) or at the ICB itself (PKiKP reflection) since we rule out heterogeneities located in the crust and/or mantle on either the source or the station side (Supplementary Info, Section 3).

The method developed in this paper independently processes the
PKP(DF) and PKiKP which allows us to separate the contribution of each phase to the residual travel times and amplitude ratios. We therefore try to identify which of the PKP(DF) or PKiKP controls the lateral variations detected with the residual travel times and amplitude ratios.

For the residual travel times, we compare the evolution in the $\delta t^{P K i K P}$ and $\delta t^{P K P(D F)}$ to the residual travel times (Fig. 13). The absolute $\delta t^{P K i K P}$ and $\delta t^{P K P(D F)}$ differential times refer to the time shift required to

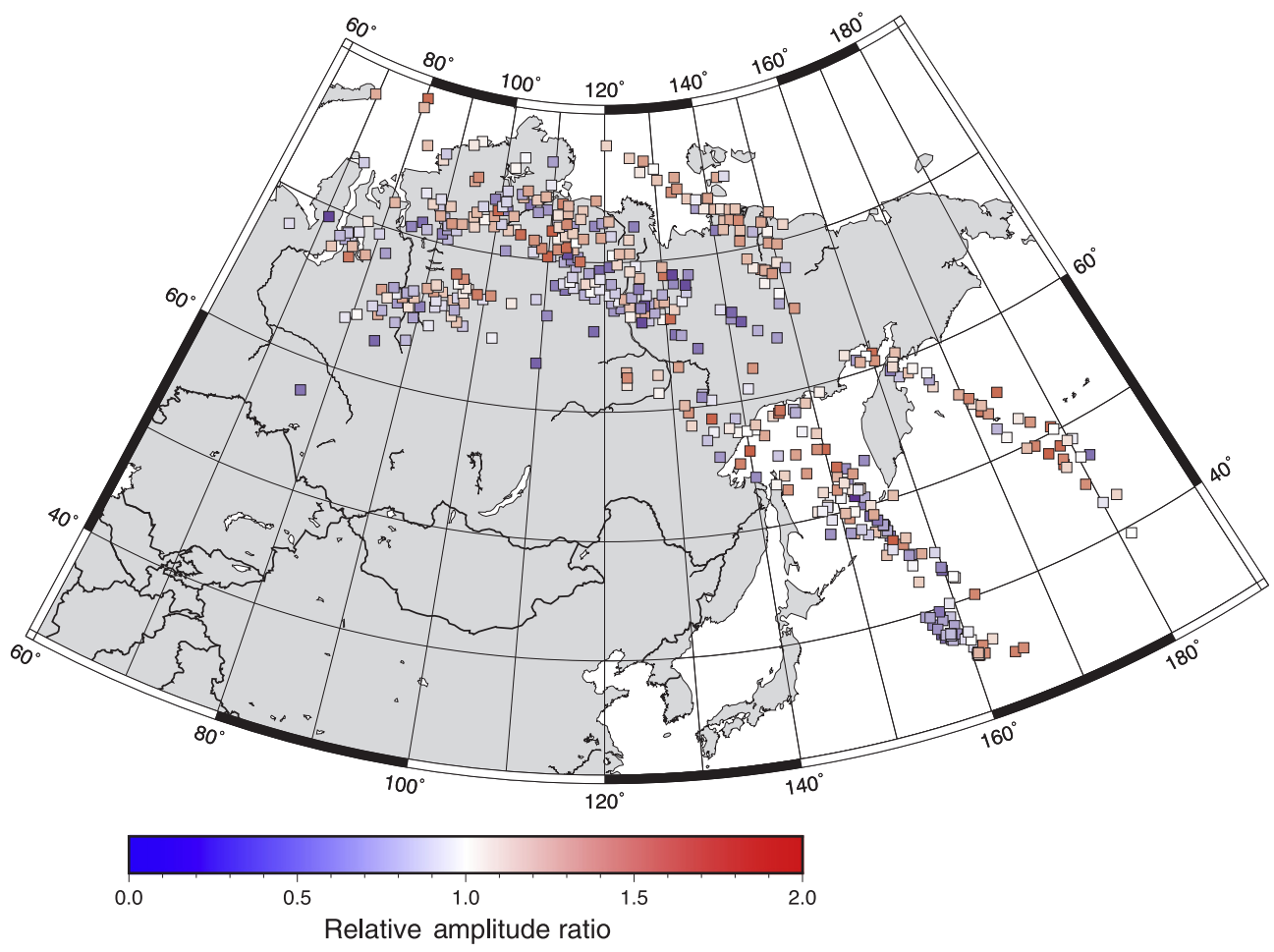

Fig. 11. Map of the relative amplitude ratios corrected for the $Q_{\alpha}$ structure inverted. 

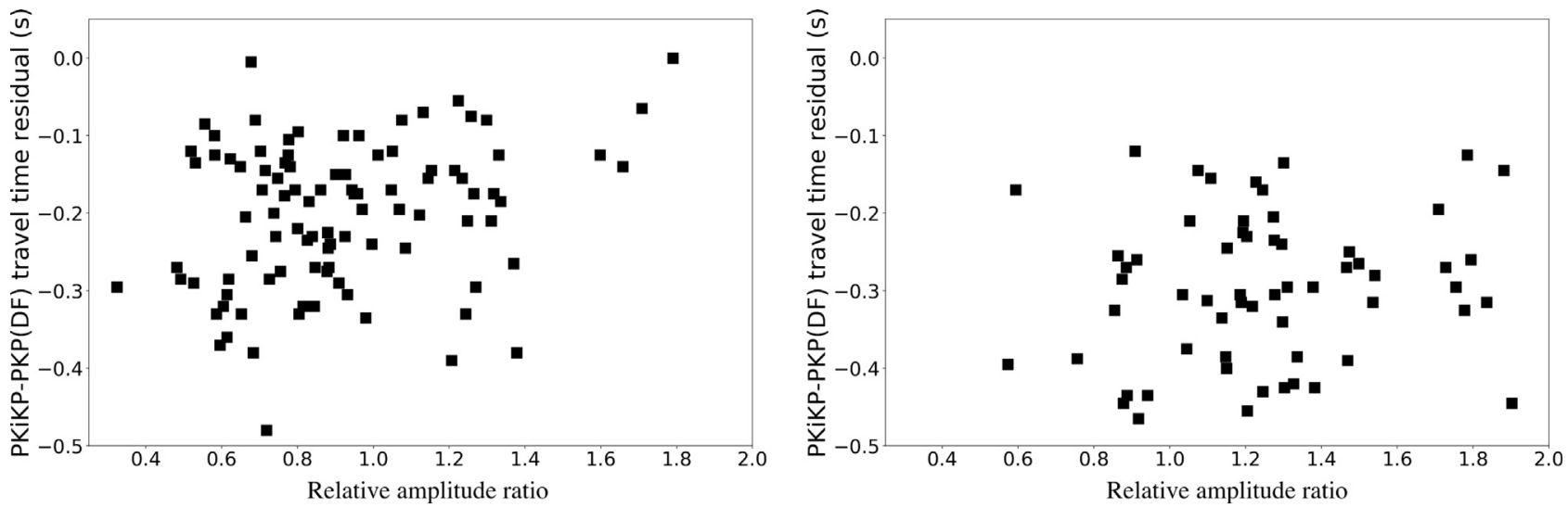

Fig. 12. Differential travel time residuals plotted with respect to relative amplitude ratios for events 201206 (left panel) and 201301 (right panel).

align the synthetic and observed waveforms of each phase. However, the two phases contribute to the residual travel times, meaning that the lateral variations detected are more likely caused by the combined effect of the ICB and uppermost inner core.

We now consider the absolute PKiKP and PKP(DF) amplitude ratios referred to AK135 as a function of the relative amplitude ratios to try and see if we can distinguish contributions from the inner core and the ICB. The PKP(DF) has also been corrected for the epicentral distance effect reported between 132 and $136^{\circ}$. Fig. 14 shows that both phases contribute to the amplitude ratios, which therefore represents the combined effect of heterogeneities at the ICB and within the inner core.

\section{Discussion and conclusion}

We have investigated lateral variations of structure in the vicinity of the ICB beneath North-Eastern Russia, using differential travel time residuals and amplitude ratios of the pair of phases PKP(DF) and PKiKP in the $132-142^{\circ}$ epicentral distance range, where PKP(DF) samples the inner core between 30 and $100 \mathrm{~km}$ depth below the ICB. A new method based on the envelope was developed to distinguish the PKP(DF) and PKiKP waveforms. The residual times and amplitude ratios could then be evaluated on a large network in order to map the short-wavelength variations beneath Eastern Russia.

Previous works on the hemispherical pattern (Tanaka and Hamaguchi, 1997; Cao and Romanowicz, 2004; Yu, 2006; Irving and Deuss, 2011) reported a higher velocity and stronger attenuation than the AK135 model in the Eastern hemisphere, which is sampled in our study. However, the residual travel times inferred with our method favor a lower velocity than in the AK135 model within the uppermost inner core. Although it disagrees with the previous descriptions of the hemispherical pattern, our results are more consistent with several

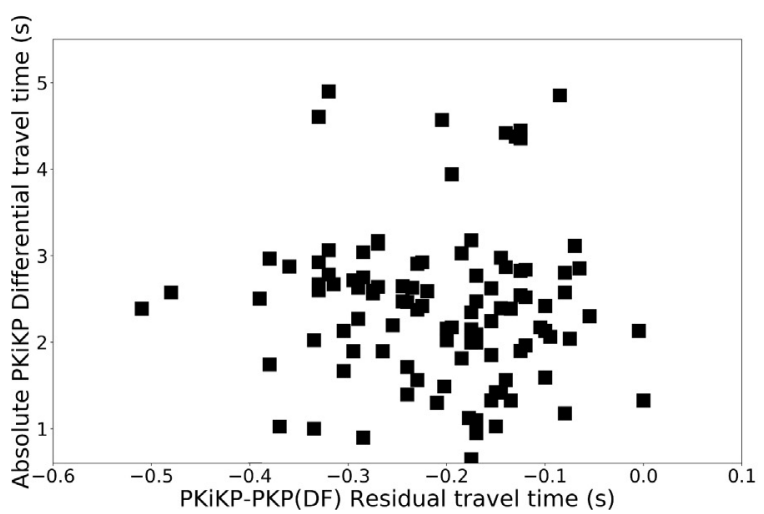

papers focusing on the North-Western Pacific (Ivan et al., 2018; Yu et al., 2017), , which both found PKiKP PKP(DF) residual travel times escillating around zero. remove « which .... around zero »

The seismic velocity of the top ten kilometers beneath the ICB is not well constrained. Godwin et al. (2018) recently proposed a velocity model in the very top of the inner core close to the region studied in this paper and suggested a velocity faster than AK135 within the uppermost $15 \mathrm{~km}$ of the inner core and a slower Vp than AK135 at greater depths. Our data are obtained between 132 and $142^{\circ}$ and are therefore more sensitive to the $30-100 \mathrm{~km}$ layer below the ICB. The low-velocity layer documented by Godwin et al. (2018) could therefore explain our observations. We therefore propose that the hemispherical boundary around $180^{\circ} \mathrm{W}$ is not well constrained in the high latitudes of the Pacific and could follow a more complex pattern instead of having a North-South orientation (Ivan et al., 2018).

We note Waszek et al. (2011) and Waszek and Deuss (2013) considered the same phase pair as in our study, and estimated differential travel time residuals and amplitude ratios for a global database. The differential travel time residuals map they obtained confirmed the East/ West dichotomy pattern with a faster Eastern hemisphere. However, their amplitude ratios map presents some variability. Several regions where fast velocity corresponds to low attenuation or the opposite indicate that, at shorter wavelengths, the velocity/attenuation correlation is not systematically respected. Likewise, Attanayake et al. (2014) reported two regions presenting a positive correlation between attenuation and velocity, but also found a zone where attenuation and velocity were anti-correlated in the Pacific, slightly to the West of our study area. However, no data sampling the high latitudes of the Western Pacific were used in this study so that this region of negative correlation might extend beneath Eastern Russia.

In 2005, Krasnoshchekov et al. (2005) proposed that the ICB is a

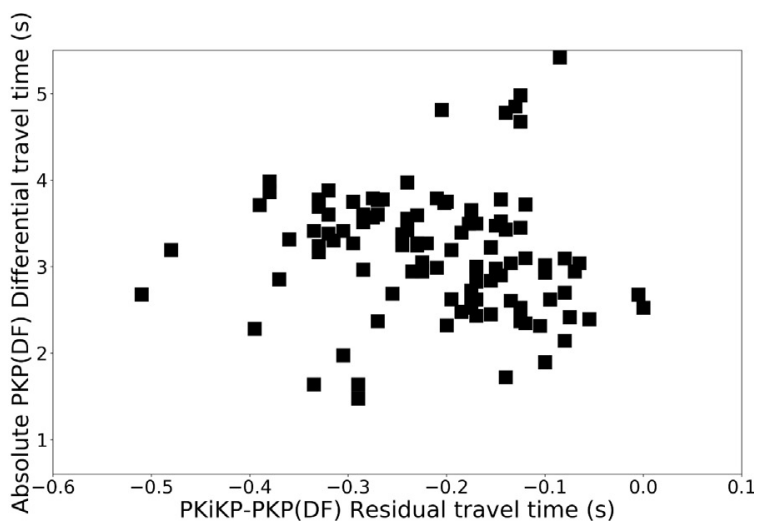

Fig. 13. Absolute PKiKP (left panel) and PKP(DF) (right panel) differential travel times plotted with respect to the residual travel times for event 201206. 

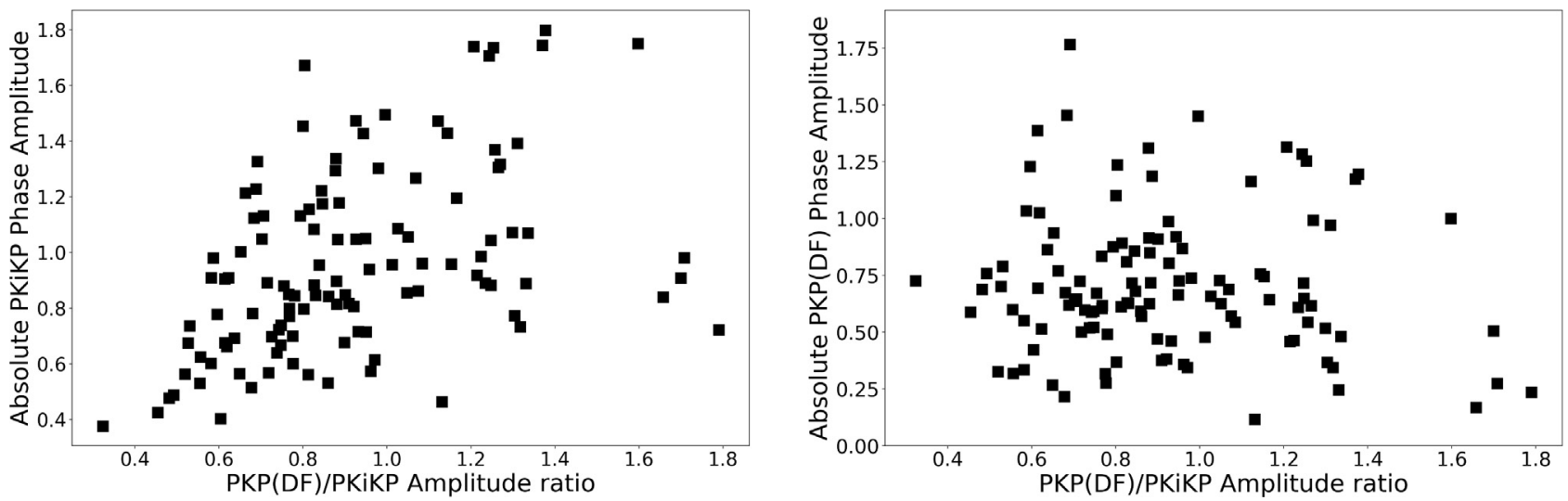

Fig. 14. PKiKP (left panel) and PKP(DF) (right panel) absolute amplitude ratio plotted with respect to the relative amplitude ratio for event 201206.

mosaic of patches with different melt content. The residual travel times and amplitude ratios map indicate that these features are $100 \mathrm{~km}$ wide, which is coherent with the heterogeneities reported by Ivan et al. (2018).

The $\mathrm{Q}_{\alpha}$ increase observed between $132^{\circ}$ and $136^{\circ}$ followed by a gentle decrease between $136^{\circ}$ and $142^{\circ}$ reflects the attenuation structure within the uppermost inner core. The corresponding $Q_{\alpha}$ versus depth profile exhibits a region of high $\mathrm{Q}_{\alpha}$ within the outermost $\sim 30 \mathrm{~km}$ of the inner core, overlying a depth interval where attenuation is strong. The presence of the high $Q_{\alpha}$ zone at the top of the inner core is in agreement with previous studies that reported the detection of such a zone at the global scale (Cao and Romanowicz, 2004; Waszek and Deuss, 2013). The deeper, low $\mathrm{Q}_{\alpha}$ layer has also been previously documented but only in the eastern hemisphere (Cao and Romanowicz, 2004; Waszek and Deuss, 2013). The thickness of this layer (15 km) is smaller, and the very low corresponding $Q_{\alpha}(\sim 30)$ more extreme than in these previous studies, but seems to be required to fit the trend in the amplitude ratio data. It is likely that the transitions from high $Q_{\alpha}$ to low $\mathrm{Q}_{\alpha}$ and back to high $\mathrm{Q}_{\alpha}$ values at depths greater than $\sim 40-45 \mathrm{~km}$ are more gradual.

The presence of a weakly attenuating zone followed by a highly attenuating layer at the top of the inner core in our region of study suggests that it reflects processes at play in the progressive solidification of the inner core, involving gradients in both melt fraction and viscosity (e.g. Singh et al., 2000). The high $Q_{\alpha}$ at the top of the inner core could indicate the presence of a mushy (Roberts et al., 2003; Bergman, 2003; Cao and Romanowicz, 2004) or slurry zone, rich in fluids. The presence of the underlying thin low $\mathrm{Q}_{\alpha}$ layer followed by high $\mathrm{Q}_{\alpha}$ is suggestive of a solid with a melt fraction that rapidly decreases with depth (Singh et al., 2000; Bergman, 2003). The varying thickness of the low $Q_{\alpha}$ zone within the eastern hemisphere and its apparent absence in the western hemisphere (Waszek and Deuss, 2013) may be significative of subtle lateral variations in temperature at the top of the inner core.

An interesting point of the method developed is the independent processing of the PKiKP and PKP(DF) waveforms which allows us to preferentially ascribe our observations to one phase and thereafter to a certain region of the core. We found that both phases were affected by the causative structure, which implies that both the ICB (or the F layer) and the structure at the top of inner core contribute to our observations. We also provided evidence that the attenuation-velocity correlation is negative beneath Eastern Russia. Therefore, the documented anti-correlation between velocity and attenuation does not hold everywhere, when looking at the regional scale.

\section{Acknowledgments}

Numerical computations were performed on the S-CAPAD platform,
IPGP, France. Figures were generated with Obspy (Beyreuther et al., 2010), Generic Mapping Tools (Wessel and Smith, 1995). We thank Editor Vernon Cormier, Annie Souriau and Satoru Tanaka for helpful reviews that improved the quality of the manuscript. This study was supported by the ERC advanced Grant WAVETOMO under the ERCs 7th Framework Program (FP7-IDEAS-ERC).

\section{Appendix A. Supplementary data}

Supplementary data associated with this article can be found, in the online version, at http://dx.doi.org/10.1016/j.pepi.2018.03.003.

\section{References}

Adam, J.-C., Romanowicz, B., 2015. Global scale observations of scattered energy near the inner-core boundary: seismic constraints on the base of the outer-core. Phys. Earth. Plan. Int. 245, 103-116.

Alboussiere, T., Deguen, R., Melzani, M., 2012. Melting-induced stratification above the earth's inner core due to convective translation. arXiv preprint arXiv:1201.1201.

Attanayake, J., Cormier, V.F., de Silva, S.M., 2014. Uppermost inner core seismic structure-new insights from body waveform inversion. Earth Plan. Sci. Lett. 385, 49-58.

Aubert, J., Amit, H., Hulot, G., Olson, P., 2008. Thermochemical flows couple the earth's inner core growth to mantle heterogeneity. Nature 454 (7205), 758.

Bergman, M., 2003. Solidification of the Earth's core. In: Earth's Core: Dynamics, Structure, Rotation Wiley Online Library, pp. 105-127.

Beyreuther, M., Barsch, R., Krischer, L., Megies, T., Behr, Y., Wassermann, J., 2010. Obspy: a python toolbox for seismology. Seismol. Res. Lett. 81 (3), 530-533.

Bullen, K., 1947. An Introduction of the Theory of Seismology. Cambridge Universty Press.

Cao, A., Masson, Y., Romanowicz, B., 2007. Short wavelength topography on the innercore boundary. Proc. Nat. Acad. Sci. 104 (1), 31-35.

Cao, A., Romanowicz, B., 2004. Hemispherical transition of seismic attenuation at the top of the earth's inner core. Earth Plan. Sci. Lett. 228 (3), 243-253.

Cleary, J., Haddon, R., 1972. Seismic wave scattering near the core-mantle boundary: a new interpretation of precursors to pkp. Nature 240 (5383), 549-551.

Cormier, V., 2007. Texture of the uppermost inner core from forward-and back-scattered seismic waves. Earth Plan. Sci. Lett. 258 (3), 442-453.

Cormier, V., Li, X., 2002. Frequency-dependent seismic attenuation in the inner core 2. A scattering and fabric interpretation. J. Geophys. Res 107 (B12).

Cormier, V., Attanayake, J., He, K., 2011. Inner core freezing and melting: constraints from seismic body waves. Phys. Earth. Plan. Int. 188 (3), 163-172.

Cormier, V., Xu, L., Choy, G., 1998. Seismic attenuation of the inner core: viscoelastic or stratigraphic? Geophys. Res. Lett. 25 (21), 4019-4022.

Côté, A., Vočadlo, L., Brodholt, J., 2008. Light elements in the core: effects of impurities on the phase diagram of iron. Geophys. Res. Lett. 35 (5).

Creager, K., 1999. Large-scale variations in inner core anisotropy. J. Geophys. Res 104 (B10), 23127-23139.

Crotwell, H., Owens, T., Ritsema, J., 1999. The taup toolkit: Flexible seismic travel-time and ray-path utilities. Seismol. Res. Lett. 70 (2), 154-160.

Cummins, P., Geller, R., Hatori, T., Takeuchi, N., 1994. Dsm complete synthetic seismograms: Sh, spherically symmetric, case. Geophys. Res. Lett. 21 (7), 533-536.

Dai, Z., Wang, W., Wen, L., 2012. Irregular topography at the earth's inner core boundary. Proc. Nat. Acad. Sci. 109 (20), 7654-7658.

De Siena, L., Del Pezzo, E., Thomas, C., Curtis, A., Margerin, L., 2013. Seismic energy envelopes in volcanic media: in need of boundary conditions. Geophys. J. Int. 195 (2), 1102-1119.

Doornbos, D., Husebye, E., 1972. Array analysis of pkp phases and their precursors. Phys. Earth. Plan. Int. 5, 387-399.

Engdahl, E., Flinn, E., Massé, R., 1974. Differential pkikp travel times and the radius of 
the inner core. Geophys. J. Int. 39 (3), 457-463.

Fearn, D., Loper, D., Roberts, P., 1981. Structure of the earth's inner core. Nature 292 (5820), 232.

Garcia, R., 2002. Constraints on upper inner-core structure from waveform inversion of core phases. Geophys. J. Int. 150 (3), 651-664.

Geballe, Z.M., Lasbleis, M., Cormier, V.F., Day, E.A., 2013. Sharp hemisphere boundaries in a translating inner core. Geophys. Res. Lett. 40, 1719-1723.

Garcia, R., Souriau, A., 2000. Inner core anisotropy and heterogeneity level. Geophys. Res. Lett. 27 (19), 3121-3124.

Geller, R., Takeuchi, N., 1995. A new method for computing highly accurate dsm synthetic seismograms. Geophys. J. Int. 123 (2), 449-470.

Godwin, H., Waszek, L., Deuss, A., 2018. Measuring the seismic velocity in the top $15 \mathrm{~km}$ of earth's inner core. Phys. Earth. Plan. Int. 274, 158-169.

Gubbins, D., Masters, G., Nimmo, F., 2008. A thermochemical boundary layer at the base of earth's outer core and independent estimate of core heat flux. Geophys. J. Int. 174 (3), 1007-1018.

Gubbins, D., Sreenivasan, B., Mound, J., Rost, S., 2011. Melting of the earth's inner core. Nature 473 (7347), 361.

Hedlin, M., Shearer, P., Earle, P., 1997. Seismic evidence for small-scale heterogeneity throughout the earth's mantle. Nature 387 (6629), 145-150.

Hibert, C., Mangeney, A., Grandjean, G., Shapiro, N., 2011. Slope instabilities in dolomieu crater, réunion island: From seismic signals to rockfall characteristics. J. Geophys. Res 116 (F4).

Irving, J., Deuss, A., 2011. Hemispherical structure in inner core velocity anisotropy. J. Geophys. Res 116 (B4).

Ivan, M., He, X., 2017. Uppermost inner core heterogeneity from differential travel times of pkikp vs. pkp-bdiff and pkp-cdiff phases. Pure Appl. Geophys. 174 (1), 249-259.

Ivan, M., Wang, R., Hofstetter, R., 2018. Non quasi-hemispherical seismological pattern of the earth's uppermost inner core. Sci. Rep. 8 (1), 2270.

Jacobs, J., 1953. The earth's inner core. Nature 172 (4372), 297-298.

Jeffreys, H., 1939. The times of the core waves. Geophys. Suppl. Mon. Not. R. Astron. Soc. 4 (8), 594-615.

Kaneshima, S., Hirahara, K., Ohtaki, T., Yoshida, Y., 1994. Seismic structure near the inner core-outer core boundary. Geophys. Res. Lett. 21 (2), 157-160.

Kennett, B., Engdahl, E., Buland, R., 1995. Constraints on seismic velocities in the earth from traveltimes. Geophys. J. Int. 122 (1), 108-124.

Koper, K., Dombrovskaya, M., 2005. Seismic properties of the inner core boundary from pkikp/p amplitude ratios. Earth Plan. Sci. Lett. 237 (3), 680-694.

Koper, K., Pyle, M., Franks, J., 2003. Constraints on aspherical core structure from pkikppcp differential travel times. J. Geophys. Res 108 (B3).

Koper, K.D., Franks, J.M., Dombrovskaya, M., 2004. Evidence for small-scale heterogeneity in earth's inner core from a global study of pkikp coda waves. Earth Plan. Sci. Lett. 228 (3), 227-241.

Krasnoshchekov, D., Kaazik, P., Ovtchinnikov, V., 2005. Seismological evidence for mosaic structure of the surface of the earth's inner core. Nature 435 (7041), 483.

Leyton, F., Koper, K.D., 2007. Using pkikp coda to determine inner core structure: 1. Synthesis of coda envelopes using single-scattering theories. J. Geophys. Res 112 (B5).

Loper, D., 1978. The gravitationally powered dynamo. Geophys. J. Int. 54 (2), 389-404.

Loper, D., Roberts, P., 1981. A study of conditions at the inner core boundary of the earth. Phys. Earth. Plan. Int. 24 (4), 302-307.

Mancinelli, N., Shearer, P., 2013. Reconciling discrepancies among estimates of smallscale mantle heterogeneity from pkp precursors. Geophys. J. Int. 195 (3), 1721-1729.

Margerin, L., Nolet, G., 2003. Multiple scattering of high-frequency seismic waves in the deep earth: Pkp precursor analysis and inversion for mantle granularity. J. Geophys. Res. 108 (B11).

Mayeda, K., Walter, W., 1996. Moment, energy, stress drop, and source spectra of western united states earthquakes from regional coda envelopes. J. Geophys. Res 101 (B5), 11195-11208.

McSweeney, T., Creager, K., Merrill, R., 1997. Depth extent of inner-core seismic anisotropy and implications for geomagnetism. Phys. Earth. Plan. Int. 101 (1-2), 131-156.

Monnereau, M., Calvet, M., Margerin, L., Souriau, A., 2010. Lopsided growth of earth's inner core. Science 328 (5981), 1014-1017.

Niu, F., Wen, L., 2001. Hemispherical variations in seismic velocity at the top of the earth's inner core. Nature 410 (6832), 1081-1084.
Niu, F., Wen, L., 2002. Seismic anisotropy in the top $400 \mathrm{~km}$ of the inner core beneath the eastern hemisphere. Geophys. Res. Lett. 29 (12).

Ohtaki, T., Kaneshima, S., 2015. Independent estimate of velocity structure of earth's lowermost outer core beneath the northeast pacific from pkikp- pkpbc differential traveltime and dispersion in pkpbc. J. Geophys. Res. 120 (11), 7572-7586.

Ohtaki, T., Kaneshima, S., Kanjo, K., 2012. Seismic structure near the inner core boundary in the south polar region. J. Geophys. Res. 117 (B3).

Roberts, P., Jones, C., Calderwood, A., 2003. Energy fluxes and ohmic dissipation in the earth's core. Earth's Core Lower Mantle 100-129.

Sato, H., 1984. Attenuation and envelope formation of three-component seismograms of small local earthquakes in randomly inhomogeneous lithosphere. J. Geophys. Res. 89 (B2), 1221-1241.

Shimizu, H., Poirier, J., Le Mouël, J., 2005. On crystallization at the inner core boundary. Phys. Earth Planet. Inter. 151 (1-2), 37-51.

Singh, S., Taylor, M., Montagner, J.-P., 2000. On the presence of liquid in earth's inner core. Science 287 (5462), 2471-2474.

Song, X., Helmberger, D., 1992. Velocity structure near the inner core boundary from waveform modeling. J. Geophys. Res 97 (B5), 6573-6586.

Souriau, A., 2007. Deep earth structure-the earth's cores. Treatise on Geophysics, 1, Seismology and Structure of the. Earth 655-693.

Souriau, A., 2015. Presumption of large-scale heterogeneity at the top of the outer core basal layer. Earth Plan. Sci. Lett. 415, 175-182.

Souriau, A., Poupinet, G., 1991. The velocity profile at the base of the liquid core from pkp (bc + cdiff) data: an argument in favour of radial inhomogeneity. Geophys. Res. Lett. 18 (11), 2023-2026.

Stroujkova, A., Cormier, V., 2004. Regional variations in the uppermost $100 \mathrm{~km}$ of the earth's inner core. J. Geophys. Res 109 (B10).

Sumita, I., Olson, P., 1999. A laboratory model for convection in earth's core driven by a thermally heterogeneous mantle. Science 286 (5444), 1547-1549.

Sun, X., Song, X., 2008. Tomographic inversion for three-dimensional anisotropy of earth's inner core. Phys. Earth. Plan. Int. 167 (1), 53-70.

Tanaka, S., Hamaguchi, H., 1997. Degree one heterogeneity and hemispherical variation of anisotropy in the inner core from pkp (bc)-pkp (df) times. J. Geophys. Res 102 (B2), 2925-2938.

Tanaka, S., Tkalčić, H., 2015. Complex inner core boundary from frequency characteristics of the reflection coefficients of pkikp waves observed by hi-net. Prog. Earth Planet. Sci. 2 (1), 34.

Vidale, J., Earle, P., 2000. Fine-scale heterogeneity in the earth's inner core. Nature 404 (6775), 273

Waszek, L., Deuss, A., 2011. Distinct layering in the hemispherical seismic velocity structure of earth's upper inner core. J. Geophys. Res 116 (B12).

Waszek, L., Deuss, A., 2013. A low attenuation layer in the earth's uppermost inner core. Geophys. J. Int. 195 (3), 2005-2015.

Waszek, L., Irving, J., Deuss, A., 2011. Reconciling the hemispherical structure of earth/'s inner core with its super-rotation. Nat. Geosci. 4 (4), 264-267.

Wen, L., 2006. Localized temporal change of the earth's inner core boundary. Science 314 (5801), 967-970.

Wen, L., Niu, F., 2002. Seismic velocity and attenuation structures in the top of the earth's inner core. J. Geophys. Res 107 (B11).

Wessel, P., Smith, W., 1995. The Generic Mapping Tools, GMT, Version 3: Technical Reference and Cookbook. School of Ocean and Earth Science and Technology, University of Hawaii at Manoa.

Yao, J., Sun, L., Wen, L., 2015. Two decades of temporal change of earth's inner core boundary. J. Geophys. Res 120 (9), 6263-6283.

Yu, W., Su, J., Song, T., Huang, H., Mozziconacci, L., Huang, B., 2017. The inner core hemispheric boundary near 180 w. Phys. Earth. Plan. Int. 272, 1-16.

Yu, W., Wen, L., 2006. Inner core attenuation anisotropy. Earth Plan. Sci. Lett. 245 (3), 581-594.

Yu, W., Wen, L., 2006. Seismic velocity and attenuation structures in the top $400 \mathrm{~km}$ of the earth's inner core along equatorial paths. J. Geophys. Res 111 (B7).

Yu, W., Wen, L., Niu, F., 2005. Seismic velocity structure in the earth's outer core. J. Geophys. Res 110 (B2)

Zou, Z., Koper, K., Cormier, V., 2008. The structure of the base of the outer core inferred from seismic waves diffracted around the inner core. J. Geophys. Res 113 (B5). 